WSRC-TR-93-064

FOURTH QUARTER 1992

AND 1992 SUMMARY

\title{
H-AREA ACID/CAUSTIC BASIN GROUNDWATER MONITORING REPORT (U)
}

PUBLICATION DATE: MARCH 1993

WESTINGHOUSE SAVANNAH RIVER COMPANY SAVANNAH RIVER SITE AIKEN, SC 29808

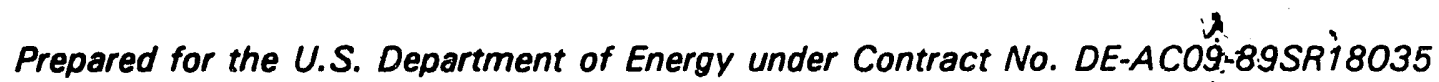




\section{DISCLAIMER}

This report was prepared by Westinghouse Savannah River Company (WSRC) for the United States Department of Energy under Contract No. DE-AC09-89SR18035 and is an account of work performed under that contract. Reference herein to any specific commercial product, process, or service by trademark, name, manufacturer, or otherwise does not necessarily constitute or imply endorsement, recommendation, or favoring of same by WSnC or by the United States Government or any agency thereof. The views and opinions of the authors expressed herein do not necessarily state or reflect those of the United States Government or any agency thereof. 


\section{FOURTH QUARTER 1992 \\ AND 1992 SUMMARY}

\section{H-AREA ACID/CAUSTIC BASIN GROUNDWATER MONITORING REPORT (U)}

KEY WORDS

HAC wells

hazardous waste

iron

specific conductance

tritium

PUBLICATION DATE: MARCH 1993

Authorized Derivative Classifier:

m.a. Etra

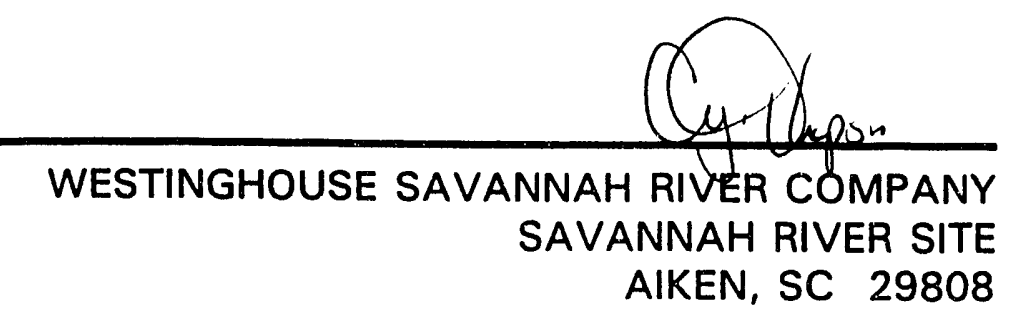




\section{Abstract}

During fourth quarter 1992, samples from the four HAC monitoring wells at the H-Area Acid/Caustic Basin received comprehensive analyses. Monitoring results that exceeded the final Primary Drinking Water Standards (PDWS) or the Savannah River Site (SRS) flagging criteria or turbidity standard during the quarter are the focus of this report.

Tritium exceeded the final PDWS in wells HAC 1, 2, 3, and 4 during fourth quarter 1992. Tritium activities in upgradient well HAC 4 were similar to tritium levels in wells HAC 1, 2, and 3 . Iron was elevated in well HAC 1,2, and 3. Specific conductance and manganese were elevated in one downgradient well each. No well samples exceeded the SRS turbidity standard.

During 1992, tritium was the only constituent that exceeded the final PDWS. It did so consistently in all four wells during all four quarters, with little variability in activity. 


\section{Contents}

Page

Abstract $\ldots \ldots \ldots \ldots \ldots \ldots \ldots \ldots \ldots \ldots \ldots \ldots \ldots \ldots \ldots \ldots \ldots \ldots \ldots \ldots$

List of Figures $\ldots \ldots \ldots \ldots \ldots \ldots \ldots \ldots \ldots \ldots \ldots \ldots \ldots \ldots$

List of Tables $\ldots \ldots \ldots \ldots \ldots \ldots \ldots \ldots \ldots \ldots \ldots \ldots \ldots \ldots \ldots$

Executive Summary $\ldots \ldots \ldots \ldots \ldots \ldots \ldots \ldots \ldots \ldots \ldots \ldots \ldots \ldots$

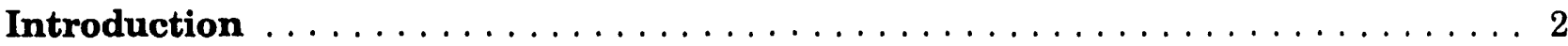

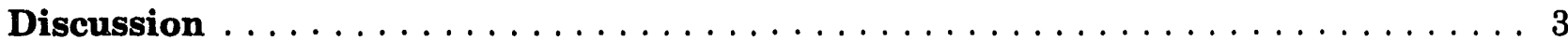

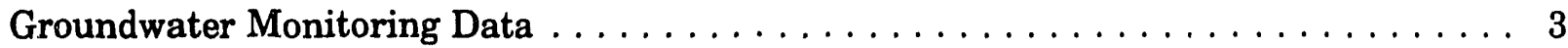

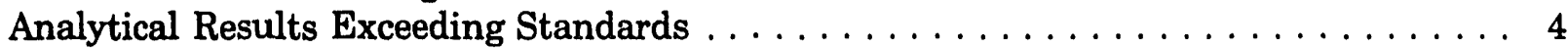

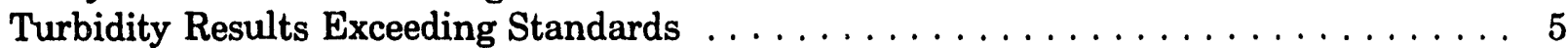

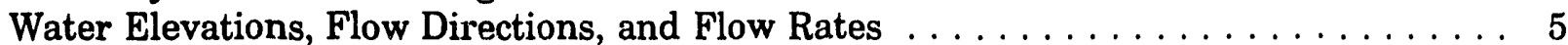

Results for Upgradient vs. Downgradient Wells $\ldots \ldots \ldots \ldots \ldots \ldots \ldots \ldots$

Conclusions $\ldots \ldots \ldots \ldots \ldots \ldots \ldots \ldots \ldots \ldots \ldots \ldots \ldots \ldots \ldots \ldots \ldots \ldots$

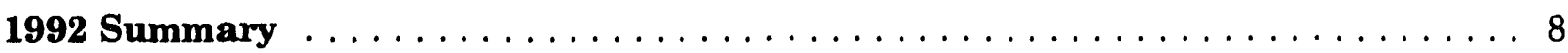

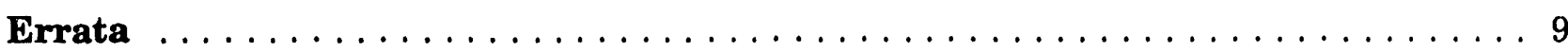

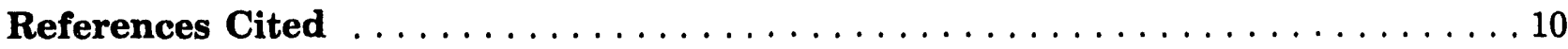

Appendix A-Final Primary Drinking Water Standards $\ldots \ldots \ldots \ldots \ldots$. $\ldots \ldots$

Appendix B-Flagging Criteria $\ldots \ldots \ldots \ldots \ldots \ldots \ldots \ldots \ldots \ldots \ldots \ldots$

Appendix C-Figures $\ldots \ldots \ldots \ldots \ldots \ldots \ldots \ldots \ldots \ldots \ldots \ldots \ldots \ldots$

Appendix D-Groundwater Monitoring Results Tables $\ldots \ldots \ldots \ldots \ldots \ldots \ldots$

Appendix E-Data Quality/Useability Assessment $\ldots \ldots \ldots \ldots \ldots \ldots \ldots$ 


\section{List of Figures}

\section{Page}

1. Location of the H-Area Acid/Caustic Basin at the Savannah River Site . . . . . . . C-2

2. Location of Groundwater Monitoring Wells at the H-Area Acid/Caustic Basin . . . . C-3

3. Water-Elevation Contour Map of the Water Table at the H-Area Acid/Caustic

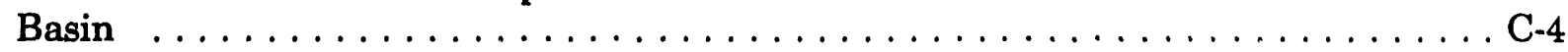

\section{List of Tables}

1. Constituents Exceeding Final Primary Drinking Water Standards for $1992 \ldots \ldots$ D-5

2. Constituents Exceeding Half the Final Primary Drinking Water Standards, Other Flag 1 or Flag 2 Criteria, or the SRS Turbidity Standard $\ldots \ldots \ldots \ldots$ D-5

3. Groundwater Monitoring Results for Individual Wells $\ldots \ldots \ldots \ldots \ldots \ldots$ D-6 
WSRC-TR-93-064 
WSRC-TR-93-064

\section{Executive Summary}

The four monitoring wells at the H-Area Acid/Caustic Basin are sampled quarterly as part of the Savannah River Site (SRS) Groundwater Monitoring Program and to comply with a consent decree executed May 26, 1988, by the U.S. District Court (District of South Carolina, Aiken Division). During fourth quarter 1992, samples from the monitoring wells received comprehensive analyses. Monitoring results that exceeded the final Primary Drinking Water Standards (PDWS), the SRS flagging criteria, or the SRS turbidity standard are the focus of this report.

During fourth quarter 1992, wells HAC 1, 2, and 3 at the H-Area Acid/Caustic Basin did not meet the criteria for purging and stabilization prior to sampling; thus, the samples collected from these wells may not be representative groundwater samples. Tritium exceeded the final PDWS in all four wells, with the highest activity $5.1 \mathrm{E}+01 \mathrm{pCi} / \mathrm{mL}$ in well HAC 1 . Iron exceeded its Flag 2 criterion in wells HAC 1, 2, and 3. Specific conductance exceeded its Flag 2 criterion in well HAC 2, and manganese exceeded its Flag 2 criterion in well HAC 3. No well samples exceeded the SRS turbidity standard.

Water-table elevations at the H-Area Acid/Caustic Basin indicate that groundwater flow direction is northwest (using SRS grid coordinates) at a rate of $580 \mathrm{ft} / \mathrm{yr}$. Tritium activities in upgradient well HAC 4 are similar to tritium levels in wells HAC 1, 2, and 3.

Tritium activities in all four wells during all four quarters were consistently above the final PDWS.

Water-elevation data from the HAC wells indicate that groundwater flow directions during 1992 were generally northwest at rates ranging from $150 \mathrm{ft} / \mathrm{yr}$ (second quarter) to $580 \mathrm{ft} / \mathrm{yr}$ (fourth quarter). 


\section{Introduction}

The H-Area Acid/Caustic Basin is southwest of the H-Area Canyon Building near the H-Area Tank Farm at the Savannah River Site (SRS). The basin, constructed in the early 1950s, is an unlined earthen pit that received dilute sulfuric acid and sodium hydroxide solutions and other wastes from several areas within SRS. The basin provided an area for the mixing and neutralization of the dilute solutions before their discharge into nearby streams. Disposal of acid/caustic solutions to the H-Area Acid/Caustic Basin was discontinued in 1982; however, the basin received steam condensate from a hose box and drainage from a chemical pad until 1985 (Heffner and Exploration Resources, 1991).

Under the terms of a consent decree executed May 26, 1988, by the U.S. District Court (District of South Carolina, Aiken Division), the basin became subject to requirements of Subtitle $\mathbf{C}$ of the Resource Conservation and Recovery Act (RCRA), the South Carolina Hazardous Waste Management Regulations (SCHWMR), and associated regulations. In the summer of 1988, a network of monitoring wells was proposed for the basin to ensure compliance with SCHWMR; in August 1988, four monitoring wells were installed at the H-Area Acid/Caustic Basin.

The monitoring wells at the H-Area Acid/Caustic Basin are sampled quarterly as part of the SRS Groundwater Monitoring Program and to comply with SCHWMR. The revised Groundwater Quality Assessment Plan (WSRC, 1991), submitted to the South Carolina Department of Health and Environmental Control on April 30,1991, indicates that the monitoring well network at the H-Area Acid/Caustic Basin is sufficient to detect any degradation of the groundwater due to past operations at the basin. 
WSRC-TR-93-064

\section{Discussion}

\section{Groundwater Monitoring Data}

The groundwater sampling procedure was modified beginning fourth quarter 1992 in response to regulatory guidance and advances in sampling equipment design (WSRC, 1992). The modified procedure requires evacuation of a minimum of two well volumes and stabilization of $\mathrm{pH}$, specific conductance, and turbidity prior to sample collection. Stability is established when a minimum of three successive measurements, taken within a given time period, are within a specified tolerance range. If a well pumps dry befure two well volumes are purged or before stabilization is achieved, it must be revisited within 24 hours for the data to be considered from a single sampling event. On the second visit within 24 hours, samples are taken without purging or stability measurements; thus, these samples may not be representative of the groundwater quality.

A further modification in the procedure is that samples collected for metals analyses are not filtered. Thus, the analyses are for total metals rather than dissolved metals. Variable-speed pumps have been installed in some vells in specific areas that have had a history of elevated metals. Samples from these wells are collected at a slower rate to minimize turbidity, which has been associated with elevated metal levels. Decreased aluminum and iron concentrations as well as lower turbidity values have been observed for samples from wells with variablespeed pumps. At present, no HAC wells have variable-speed pumps.

During fourth quarter 1992, samples from four monitoring wells at the H-Area Acid/Caustic Basin received comprehensive analyses. This report describes monitoring results that exceeded the Safe Drinking Watur Act final Primary Drinking Water Standards (PDWS) or screening levels, set by the U.S. Environmental Protection Agency (EPA) (Appendix A), the South Carolina final Primary Drinking Water Standard for lead (Appendix A), other SRS flagging criteria (Appendix B), or the SRS turbidity standard.

The drinking water standard for lead was changed to the South Carolina Primary Drinking Water Standard of $50 \mu \mathrm{g} / \mathrm{L}$ fourth quarter 1992. Lead data for the earlier quarters of 1992 were made consistent with the $50 \mu \mathrm{g} / \mathrm{L}$ standard for this annual report. The SRS flagging criteria are based on final and proposed PDWS, Secondary Drinking Water Standards, and method detection limits. For simplicity, results that either equal or exceed standards are described only as exceeding or above standards. Constituent levels that exceed the final PDWS, screening levels, or Flag 2 criteria are described as elevated, and constituent levels that exceed Flag 1 criteria are described as slightly elevated.

The final PDWS for individual analytes provided in Appendix A may not always match the SRS flagging criteria provided in Appendix B. The final PDWS are used as guidelines in this compliance report to meet regulatory requirements; the flagging criteria are used by 
EPD/EMS to identify relative levels of constituents in the groundwater and as guides for scheduling groundwater sampling.

Appendix $\mathbf{C}$ presents illustrations of the monitored waste management unit at SRS (Figure 1), the individual monitoring wells (Figure 2), and the flow directions of the groundwater beneath the basin (Figure 3). Monitoring results as well as analyses that exceeded holding times, the final PDWS, other flagging criteria, or the turbidity standard are presented in Appendix D; and a discussion of data quality and useability is in Appendix E.

\section{Analytical Results Exceeding Standards}

Results for analytes that exceeded the final PDWS (see Aprendix A) during fourth quarter 1992 are summarized in Table 1 (Appendix D). Wells HAC 1, 2, 3, and 4 all contained tritium activities that exceeded the final PDWS, with values ranging up to $5.1 \mathrm{E}+01 \mathrm{pCi} / \mathrm{mL}$ in well HAC 1.

Constituents that exceeded other Flag 1 and 2 criteria (see Appendix B) during fourth quarter 1992 are summarized in Table 2 (Appendix D). Iron exceeded the Flag 2 criterion in wells HAC 1,2 , and 3, with a maximum concentration of $2,070 \mu \mathrm{g} / \mathrm{L}$ in well HAC 2 . Specific conductance exceeded its Flag 2 criterion in well HAC 2, and manganese exceeded its Flag 2 criterion in well HAC 3.

Table 3 (Appendix D) presents all of the results for individual wells and indicates those analyses that exceeded holding times and the final PDWS. Table 3 also lists the number of well volumes of water purged from each well during fourth quarter 1992 at the H-Area Acid/Caustic Basin. Wells HAC 1, 2, and 3 each failed to meet the new criteria for purging and stabilization prior to purging dry. Each was sampled later the same day. The samplers noted that the water from HAC 3 was a very light brown.

Constituent results are compared with the PDWS in the database of values reported by the laboratory. Many constituents are reported to more significant digits in the database than in these reports. Thus, some constituent results in Table 3 that appear to equal the PDWS are not marked in the $D$ column. Those results are below the PDWS in the database.

Some of the values for earlier quarters presented in the results tables of this rejort may differ from the values for those same quarters presented in previous reports, arid reported values may not match reported sample dates. These differences result from the following: (1) the computer program that creates the analytical results tables was revised beginning second quarter 1992 to present the highest value for analytes with more than one result (previously, the program presented the first value encountered in the database); (2) a new computer program, which rounds numbers differently from the former computer program, was first used during third quarter 1992; and (3) some reanalyses may have been performed by the laboratories after the quarterly reports had gone to press. The sample dates in Table 3 are the dates when the field data were collected. These dates may differ from the dates of the laboratory analyses if the highest results were obtained for samples collected on different dates. 


\section{Turbidity Results Exceeding Standards}

Turbidity results, in nephelometric turbidity units (NTU), are reported for well samples exceeding 5 NTU. During fourth quarter 1992, wells HAC 1, 2, and 3 had turbidity values between 5 NTU and the SRS turbidity standard of 50 NTU (Table 3, Appendix D).

The value of 5 NTU, established by EPA (1986) as a general stanaard for acceptability of groundwater samples, is considered unrealistic for monitoring wells at SRS. Gass (1989) has documented turbidity measurements ranging up to 5,000 NTU from properly designed wells screened in poorly productive formations, such as those screened in the water table. During the 1989 RCRA Compliance Evaluation Inspection, officials from EPA Region IV indicated that the SRS turbidity standard of 50 NTU is conservative. These officials also agreed that water-table wells in this area often correspond to nonaquifer formations, rendering development of these wells more difficult due to the low yield and high proportion of mobile fines typical of these formations (Bergren and Bennett, 1989).

\section{Water Elevations, Flow Directions, and Flow Rates}

Water-table elevations and the groundwater flow direction beneath the H-Area Acid/Caustic Basin (using SRS grid coordinates) are shown in Figure 3 (Appendix C). The horizontal gradient at the H-Area Acid/Caustic Basin is very low. However, the availability of water elevations from five nearby wells of the HTF series facilitated the determination of local flow direction. The northwest groundwater flow direction determined from this quarter's waterlevel elevations for wells HAC $1,2,3$, and 4 and HTF $13,14,15,16,18,19,20$, and 21 is consistent with the historical flow pattern. Although HTF 15, 18, and 19 are outside the frame of Figure 3, they were used in contouring.

The groundwater flow rate in the water table (Aquifer Zone $\mathrm{IIB}_{2}$ ) beneath the H-Area Acid/Caustic Basin is estimated using the following equation:

$$
\text { Flow }(\mathrm{ft} / \text { day })=\frac{\text { Hydraulic Conductivity }(\mathrm{ft} / \text { day) }}{\text { Porosity (unitless) }} \times \frac{d h(\mathrm{ft})}{d l(\mathrm{ft})}
$$

A hydraulic conductivity constant of $10 \mathrm{ft} /$ day (Geraghty \& Miller, 1990) is used as a conservative estimate (i.e., the actual hydraulic conductivity should be somewhat less than $10 \mathrm{ft} /$ day). The effective porosity value is estimated at $20 \%$ (Killian et al., 1987), $d h$ is the difference in head, and $d l$ is the length of the flow path. Flow rate estimates vary depending on the hydraulic gradient between wells, the size of the area under consideration, and the number of data points. For this reason, the estimation of flow rate should be considered accurate to an order of magnitude only.

Flow rate estimates are calculated as follows: flow path length is calculated to the nearest $10 \mathrm{ft}$. Flow rate per day is calculated to two significant figures using the above equation. This value is then multiplied by 365 and rounded to two significant figures for the flow rate per year. 
Using the above equation with $d h=12 \mathrm{ft}$ and $d l=371$ ) $\mathrm{ft}$ (see Figure 3 in Appendix C), the flow rate estimate for groundwater in the water table beneath the H-Area Acid/Caustic Basin is as follows:

$$
\begin{aligned}
& \frac{10}{0.20} \times \frac{12}{370}=1.6 \mathrm{ft} / \text { day } \\
& 1.6 \mathrm{ft} / \text { day } \times 365 \text { days } \approx 580 \mathrm{ft} / \mathrm{yr}
\end{aligned}
$$

\section{Results for Upgradient vs. Downgradient Wells}

Well HAC 4 is the designated upgradient well at the H-Area Acid/Caustic Basin. During fourth quarter 1992, tritium activities exceeded the final PDWS in both upgradient and downgradient wells at this waste management unit, with values up to $5.1 \mathrm{E}+01 \mathrm{pCi} / \mathrm{mL}$ in well HAC 1. Iron exceeded the Flag 2 criterion in wells $\mathrm{HAC} 1,2$, and 3 . Iron and specific conductance each exceeded the Flag 2 criterion in one downgradient well. 


\section{Conclusions}

Tritium activities exceeded the final PDWS during fourth quarter 1992 in wells HAC 1, 2, 3, and 4 at the $\mathrm{H}$-Area Acid/Caustic Basin, with activities ranging up to $5.1 \mathrm{E}+01 \mathrm{pCi} / \mathrm{mL}$ in well HAC 1. Tritium activities in upgradient well HAC 4 are similar to tritium activities in wells HAC 1, 2, and 3. Because historical records indicate that no radionuclides were disposed of at this waste management unit (Heffner and Exploration Resources, 1991), the elevated levels of tritium in the HAC wells are not considered a result of seepage from the basin. Releases of tritium from other facilities in $\mathrm{F}$ and $\mathrm{H}$ areas, including the high-level waste tank farm adjacent to the H-Area Acid/Caustic Basin, are possible sources of the tritium.

Iron exceeded the Flag 2 criterion in downgradient wells HAC 1, 2, and 3. The marked increase in fourth quarter iron concentrations in those three wells is concurrent with the change to analysis of total metals rather than dissolved metals. Specific conductance exceeded its Flag 2 criterion in well HAC 2, and manganese exceeded its Flag 2 criterion in well HAC 3. Generally, elevated levels of constituents found in downgradient wells but not in upgradient wells at a waste management unit are considered products of the waste management unit.

No well samples exceeded the 50 NTU SRS turbidity standard.

Water-table elevations at the H-Area Acid/Caustic Basin indicate that groundwater flow is toward the northwest relative to SRS grid coordinates at a rate of $580 \mathrm{ft} / \mathrm{yr}$; this flow direction is consistent with the historical flow pattern. The revised Groundwater Quality Assessment Plan (WSRC, 1991) for the unit provides evidence that wells HAC 1, 2, and 3 are consistently downgradient of well HAC 4 and that the monitoring well network is sufficient to detect degradation of the groundwater due to past operations at the basin. 


\section{Summary}

Tritium was the only constituent detected above final PDWS in the HAC wells during 1992. Activities were similar in all four wells during all four quarters, ranging only from a low in well HAC 4 during first quarter of $2.7 \mathrm{E}+01$ to a high of $5.3 \mathrm{E}+01$ in well HAC 1 during both first and third quarters. Tritium consistently exceeded its PDWS in all four of the HAC wells during 1991 , with levels ranging from $3.0 \mathrm{E}+01 \mathrm{pCi} / \mathrm{mL}$ in well $\mathrm{HAC} 4$ to $6.2 \mathrm{E}+01 \mathrm{pCi} / \mathrm{mL}$ in well HAC 1. An elevated level of total alpha-emitting radium was detected in well HAC 2 during third quarter 1991.

A result for radium-228 above the final PDWS for total radium (radium-226 plus radium-228) during third quarter 1992 was not supported by other analyses during the year.

Water-elevation data from the HAC wells indicate that groundwater flow directions during 1992 were generally northwest at rates ranging from $150 \mathrm{ft} / \mathrm{yr}$ (second quarter) to $580 \mathrm{ft} / \mathrm{yr}$ (fourth quarter). Flow in 1991 was to the northwest at rates ranging from approximately $110 \mathrm{ft} / \mathrm{yr}$ to $290 \mathrm{ft} / \mathrm{yr}$. 


\section{Errata}

First Quarter 1992:

- The definitions for the abbreviations $B A$ and $W A$ were not included in the "Key to Reading the Tables" in Appendix D. BA and WA represent Barringer Laboratories, Inc., and Roy F. Weston, Inc., laboratories, respectively.

Second Quarter 1992:

- No errata have been reported.

Third Quarter 1992:

- Prior to third quarter 1992, the results of certain analyses for nitrate-nitrite as nitrogen were reported incorrectly by the General Engineering laboratory as nitrate as nitrogen results. The analyses in the results tables of this report are reported correctly (nitratenitrite results have been separated from true nitrate results). 


\section{References Cited}

Bergren, C. L., and C. B. Bennett, 1989. Assessment of SRS Groundwater Monitoring Wells Impacted by Turbidity, WSRC-RP-89-891. Westinghouse Savannah River Company. Aiken, SC.

EPA (U.S. Environmental Protection Agency), 1986. RCRA Ground Water Monitoring Technical Enforcement Guidance Document, OSWER-9950.1. Washington, DC.

Gass, T. E., 1989. Monitoring Wells in Non-Aquifer Formations. Water Well Journal 43(2):27-29.

Geraghty \& Miller, Inc., 1990. Evaluation of Integrated Waste Facility Closure Capping on Ground-Water Flow and Solute Transport in General Separations Area, Savannah River Site: Flow Model and Particle-Tracking Analysis, Final Report.

Prepared by Geraghty \& Miller Modeling Group for Westinghouse Savannah River Company, Waste Management Technology, Savannah River Site, Aiken, SC.

Heffner, J. D., and Exploration Resources, Inc., 1991. Technical Summary of Groundwater Quality Protection Program at the Savannah River Site (1952-1986), Volume I-Site Geohydrology and Waste Sites, DPSP-88-1002. Westinghouse Savannah River Company, Aiken, SC.

Killian, T. H., N. L Kolb, P. Corbo, and I. W. Marine, 1987. F-Area Seepage Basins, DPST-85-704. Savannah River Laboratory, E. I. du Pont de Nemours \& Company, Aiken, SC.

WSRC (Westinghouse Savannah River Company), 1991. F-, H-, K-, and P-Area Acid/Caustic Basins Groundwater Quality Assessment Plan, WSRC-TR-91-178, Revision 1.0. Westinghouse Savannah River Company, Aiken, SC.

WSRC, 1992. Hydrogeologic Data Collection Procedures and Specifications: Sampling Groundwater Monitoring Wells, Manual 3Q5, Chapter 14, Revision 0. Environmental Protection Department, Environmental Monitoring Section, Savannah River Site, Aiken, SC. 
WSRC-TR-93-064

Appendix A - Final Primary Drinking Water Standards 


\section{Final Primary Drinking Water Standards}

\begin{tabular}{|c|c|c|c|c|}
\hline Analyte & Unit & Level & $\underline{\text { Status }}$ & Reference \\
\hline Arsenic & $\mu g / L$ & 50 & Final & CFR, 1991 \\
\hline Barium & $\mu g / L$ & 2,000 & Final & CFR, 1991 \\
\hline Benzene & $\mu g / L$ & 5 & Final & CFR, 1991 \\
\hline Bromodichloromethane & $\mu g / L$ & $100^{a}$ & Final & CFR, 1991 \\
\hline Bromoform & $\mu g / L$ & $100^{a}$ & Final & CFR, 1991 \\
\hline Cadmium & $\mu g / L$ & 5 & Final & CFR, 1991 \\
\hline Carbon tetrachloride & $\mu g / L$ & 5 & Final & CFR, 1991 \\
\hline Chlordane & $\mu g / L$ & 2 & Final & CFR, 1991 \\
\hline Chloroethene (Vinyl chloride) & $\mu g / L$ & 2 & Fina! & CFR, 1991 \\
\hline Chloroform & $\mu g / L$ & $100^{a}$ & Final & CFR, 1991 \\
\hline Chromium & $\mu g / L$ & 100 & Final & CFR, 1991 \\
\hline Copper & $\mu g / L$ & 1,300 & Final & CFR, 1991 \\
\hline Dibromochloromethane & $\mu g / L$ & $100^{a}$ & Final & CFR, 1991 \\
\hline Dibromochloropropane & $\mu g / L$ & 0.2 & Final & CFR, 1991 \\
\hline 1,2-Dichlorobenzene & $\mu g / L$ & 600 & Final & CFR, 1991 \\
\hline 1,4-Dichlorobenzene & $\mu g / L$ & 75 & Final & CFR, 1991 \\
\hline 1,2-Dichloroethane & $\mu \mathrm{g} / \mathrm{L}$ & 5 & Final & CFR, 1991 \\
\hline 1,1-Dichloroethylene & $\mu g / L$ & 7 & Final & CFR, 1991 \\
\hline cis-1,2-Dichloroethylene & $\mu g / L$ & 70 & Final & CFR, 1991 \\
\hline trans-1,2-Dichloroethylene & $\mu \mathrm{g} / \mathrm{L}$ & 100 & Final & CFR, 1991 \\
\hline 2,4-Dichlorophenoxyacetic acid & $\mu g / L$ & 70 & Final & CFR, 1991 \\
\hline 1,2-Dichloropropane & $\mu \mathrm{g} / \mathrm{L}$ & 5 & Final & CFR, 1991 \\
\hline Endrin & $\mu \mathrm{g} / \mathrm{L}$ & 0.2 & Final & CFR, 1991 \\
\hline Ethylbenzene & $\mu \mathrm{g} / \mathrm{L}$ & 700 & Final & CFR, 1991 \\
\hline Fluoride & $\mu g / L$ & 4,000 & Final & CFR, 1991 \\
\hline Gross alphab & $\mathrm{pCi} / \mathrm{L}$ & $1.5 E+01$ & Final & CFR, 1991 \\
\hline Heptachlor & $\mu g / L$ & 0.4 & Final & CFR, 1991 \\
\hline Heptachlor epoxide & $\mu g / L$ & 0.2 & Final & CFR, 1991 \\
\hline Lead & $\mu g / L$ & 50 & Final & $\begin{array}{l}\text { SCDHEC, } \\
1981\end{array}$ \\
\hline Lindane & $\mu g / L$ & 0.2 & Final & CFR, 1991 \\
\hline Mercury & $\mu g / L$ & 2 & Final & CFR, 1991 \\
\hline Methoxychlor & $\mu \mathrm{g} / \mathrm{L}$ & 40 & Final & CFR, 1991 \\
\hline Nitrate as nitrogen & $\mu g / L$ & 10,000 & Final & CFR, 1991 \\
\hline Nitrate-nitrite as nitrogen & $\mu \mathrm{g} / \mathrm{L}$ & 10,000 & Final & CFR, 1991 \\
\hline Nitrite as nitrogen & $\mu g / L$ & 1.000 & Final & CFR, 1991 \\
\hline Nonvolatile betac & $\mathrm{pCi} / \mathrm{L}$ & $5 E+01$ & Final & EPA, 1977 \\
\hline $\mathrm{PCBs}^{\mathrm{d}}$ & $\mu g / L$ & 0.5 & Final & CFR, 1991 \\
\hline Pentachlorophenol & $\mu \mathrm{g} / \mathrm{L}$ & 1 & Final & CFR, 1991 \\
\hline Selenium & $\mu \mathrm{g} / \mathrm{L}$ & 50 & Final & CFR, 1991 \\
\hline Strontium-89/90 & $\mathrm{pCi} / \mathrm{L}$ & $8 E+00$ & Final & CFR, 1991 \\
\hline Strontium-90 & $\mathrm{pCi} / \mathrm{L}$ & $8 E+00$ & Final & CFR, 1991 \\
\hline Styrene & $\mu g / L$ & 100 & Final & CFR, 1991 \\
\hline Tetrachloroethylene & $\mu g / L$ & 5 & Final & CFR, 1991 \\
\hline Toluene & $\mu \mathrm{g} / \mathrm{L}$ & 1,000 & Final & CFR, 1991 \\
\hline Total radium (Radium-226 and -22.8 ) & $\mathrm{pCi} / \mathrm{L}$ & $5 E+00$ & Final & CFR, 1991 \\
\hline Total trihalomethanes & $\mu g / L$ & 100 & Final & CFR, 1991 \\
\hline Toxaphene & $\mu g / L$ & 3 & Final & CFR, 1991 \\
\hline 2,4,5-TP (Silvex) & $\mu \mathrm{g} / \mathrm{L}$ & 50 & Final & CFR, 1991 \\
\hline $1,1,1$-Trichloroethane & $\mu g / L$ & 200 & Final & CFR, 1991 \\
\hline
\end{tabular}


Analyte

Trichloroethylene

Tritium

Xylenes
Unit

$\mu g / L$

$\mathrm{pCi} / \mathrm{mL}$

$\mu \mathrm{g} / \mathrm{L}$
Level

\section{5}

$2 E+01$

10,000
Status

Final

Final

Final
Reference

CFR, 1991

CFR, 1991

CFR, 1991

Note: The drinking water standard for lead was changed to the South Carolina Primary Drinking Water Standard of $50 \mu \mathrm{g} / \mathrm{L}$ fourth quarter 1992.

a This value is the drinking water standard for total trihalomethanes the sum of bromoform, bromodichloromethane, chloroform, and dibromochloromethane).

b The standard given is for gross alpha including radium-226 but excluding radon and uranium.

c This is the screening level above which providers of public drinking water should perform analyses for specific man-made radionuclides. The standard for the total dose equivalent from all such radionuclides is $4 \mathrm{mrem}$ per year.

d Analyses were conducted in 1992 for the following: PCB 1016, PCB 1221, PCB 1232, PCB 1242, PCB 1248, PCB 1254, and PCB 1260.

- For double radionuclide analyses where each separate radionuclide has its own standard, the more stringent standard is used.

\section{References}

CFR (Code of Federal Regulations), 1991. National Primary Drinking Water Regulations, 40 CFR, Part 141, pp. 578-715. Washington, DC.

EPA (U.S. Environmental Protection Agency), 1977. National Interim Primary Drinking Water Regulations, EPA-570/9-76-003. Washington, DC.

SCDHEC (South Carolina Department of Health and Environmental Control), 1981. State Primary Drinking Water Regulations, R.61-58.5. Columbia, SC. 
WSRC-TR-93-064 
WSRC-TR-93-064

Appendix B - Flagging Criteria 


\section{Flagging Criteria}

Beginning in 1991, the Savannah River Site Environmental Protection Department/ Environmental Monitoring Section modified its guidelines for flagging constituents in the Groundwater Monitoring Program. These flagging criteria are as follows:

- Flag 2 criteria for constituents equal the Safe Drinking Water Act (SDWA) final Primary Drinking Water Standard (PDWS), the SDWA proposed PDWS, or the SDWA Secondary Drinking Water Standard (SDWS). If a constituent does not have a drinking water standard, the Flag 2 criterion equals 10 times the method detection limit (MDL) calculated as the 90 th percentile detection limit obtained recently by one of the primary analytical laboratories.

- Flag 1 criteria for constituents equal one-half of the final PDWS, one-half the proposed PDWS, or one-half the SDWS. If a constituent does not have an drinking water standard, the Flag 1 criterion equals 5 times the MDL calculated as the 90th percentile detection limit obtained recently by one of the primary analytical laboratories.

- Flag 0 criteria are assigned to constituent levels below Flag 1 criteria, constituent levels below the sample detection limits, or constituents having no flagging criteria.

The following parameters are not assigned flagging criteria: alkalinity, calcium, carbonate, color, corrosivity, magnesium, odor, potassium, Eh, silica, sodium, total dissolved solids, total phosphorus, total phosphates (as P), and turbidity. In addition, common laboratory contaminants and cleaners including phthalates, methylene chloride, ketones, and toluene are not assigned flagging criteria.

\begin{tabular}{|c|c|c|c|c|}
\hline Analyte & $\underline{\text { Unit }}$ & Flag 1 & Flag 2 & Source \\
\hline Acenaphthene & $\mu \mathrm{g} / \mathrm{L}$ & 50 & 100 & EPA Method 8270 \\
\hline Acenaphthylene & $\mu g / L$ & 50 & 100 & EPA Method 8270 \\
\hline Acetone & $\mu g / L$ & 50 & 100 & EPA Metnod 8240 \\
\hline Acetonitrile (Methyl cyanide) & $\mu \mathrm{g} / \mathrm{L}$ & 500 & 1,000 & EPA Method 8240 \\
\hline Acetophenone & $\mu \mathrm{g} / \mathrm{L}$ & 50 & 100 & EPA Method 8270 \\
\hline 2-Acetylaminofluorene & $\mu g / L$ & 50 & 100 & EPA Method 8270 \\
\hline Acrolein & $\mu g / L$ & 100 & 200 & EPA Method 8240 \\
\hline Acrylonitrile & $\mu g / L$ & 100 & 200 & EPA Method 8240 \\
\hline Aldrin & $\mu \mathrm{g} / \mathrm{L}$ & 2.5 & 5 & EPA Method 8080 \\
\hline Alkalinity (as $\mathrm{CaCO}_{3}$ ) & & No flag & No flag & Set by EPD/EMS \\
\hline Allyl chloride & $\mu g / L$ & 250 & 500 & EPA Method 8240 \\
\hline Aluminum & $\mu g / L$ & 25 & 50 & Secondary DWS (CFR, 1991b) \\
\hline Americium-241 & $\mathrm{pCi} / \mathrm{L}$ & $3.17 E+00$ & $6.34 E+00$ & Proposed DWS (ErA, 1991) \\
\hline Americium-243 & $\mathrm{pCi} / \mathrm{L}$ & $3.19 E+00$ & $6.37 E+00$ & Proposed DWS (EPA, 1991) \\
\hline 4-Aminobiphenyl & $\mu g / L$ & 50 & 100 & EPA Method 8270 \\
\hline Ammonia & $\mu \mathrm{g} / \mathrm{L}$ & 500 & 1,000 & APHA Method 417B \\
\hline Ammonia nitrogen & $\mu g / L$ & 50 & 100 & EPA Method 350.1 \\
\hline Aniline & $\mu \mathrm{g} / \mathrm{L}$ & 50 & 100 & EPA Method 8270 \\
\hline Anthracene & $\mu \mathrm{g} / \mathrm{L}$ & 50 & 100 & EPA Method 8270 \\
\hline Antimony & $\mu \mathrm{g} / \mathrm{L}$ & 2.5 & 5 & Proposed DWS (EPA, 1990) \\
\hline Antimony-125 & $\mathrm{pCi} / \mathrm{L}$ & $1.5 E+02$ & $3 E+02$ & Final DWS (EPA, 1977) \\
\hline Aramite & $\mu \mathrm{g} / \mathrm{L}$ & 50 & 100 & EPA Method 8270 \\
\hline
\end{tabular}




\begin{tabular}{|c|c|c|c|c|}
\hline Analyte & Unit & Flag 1 & Flag 2 & Source \\
\hline Arsenic & $\mu g / L$ & 25 & 50 & Final DWS (CFR, 1991a) \\
\hline Barium & $\mu g !:$ & 1,000 & 2,000 & Final DWS (CFR, 1991a) \\
\hline Barium-140 & $\mathrm{pC} / \mathrm{L}$ & $4.5 E+01$ & $9 E+01$ & Final DWS (EPA, 1977) \\
\hline Benzene & $\mu g / L$ & 2.5 & 5 & Final DWS (CFR, 1991a) \\
\hline alpha-Benzene hexachloride & $\mu g / L$ & 2.5 & 5 & EPA Method 8080 \\
\hline beta-Benzene hexachloride & $\mu g / L$ & 2.5 & 5 & EPA Method 8080 \\
\hline delta-Benzene hexachloride & $\mu g / L$ & 2.5 & 5 & EPA Method 8080 \\
\hline Benzidine & $\mu g / L$ & 250 & 500 & EPA Method 8270 \\
\hline Benzola]anthracene & $\mu g / L$ & 0.05 & 0.1 & Proposed DWS (EF.A, 1990) \\
\hline Benzolb]fluoranthene & $\mu g / L$ & 0.1 & 0.2 & Proposed DWS (EPA, 1990) \\
\hline Benzolk]fluoranthene & $\mu g / L$ & 0.1 & 0.2 & Proposed DWS (EPA, 1990) \\
\hline Benzo[g,h,i]perylene & $\mu g / L$ & 50 & 100 & EPA Method 8270 \\
\hline Benzolalpyrene & $\mu g / L$ & 0.1 & 0.2 & Proposed DWS (EPA, 1990) \\
\hline Benzoic acid & $\mu g / L$ & 250 & 500 & EPA Method 8270 \\
\hline 1,4-Benzoquinone & $\mu \mathrm{g} / \mathrm{L}$ & 50 & 100 & EPA Method 8270 \\
\hline Benzyl alcohol & $\mu g / L$ & 100 & 200 & EPA Method 8270 \\
\hline Beryllium & $\mu g / L$ & 0.5 & 1 & Proposed DWS (EPA, 1990) \\
\hline Beryllium-7 & $\mathrm{pCi} / \mathrm{L}$ & $3 E+03$ & $6 E+03$ & Final DWS (EPA, 1977) \\
\hline Bis(2-chloroethoxy) methane & $\mu g / L$ & 50 & 100 & EPA Nethod 8270 \\
\hline Bis(2-chloroethyl) ether & $\mu g / L$ & 50 & 100 & EPA Method 8270 \\
\hline Bis (2-chloroisopropyl) ettier & $\mu g / L$ & 50 & 100 & EPA Method 8270 \\
\hline Bis(chloromethyl) ether & I/G & 50 & 100 & EPA Method 8270 \\
\hline Bis(chloromethyl-ethyl) ether & $\mu g / L$ & 50 & 100 & EPA Method 8270 \\
\hline Bis(2-ethylhexyl) phthalate & & No flag & No flag & Set by EPD/EMS \\
\hline Bromide & $\mu g / L$ & 5,000 & 10,000 & EPA Method 300.0 \\
\hline Bromodichloromethane & $\mu g / 1$ & 50 & 100 & Final DWS (CFR, 1991a) \\
\hline Bromoform & $\mu g / L$ & 50 & 100 & Final DWS (CFR, 1991a) \\
\hline Bromomethane (Merhyl bromide) & $\mu g / L$ & 5 & 10 & EPA Method 82.40 \\
\hline 4-Bromophenyl phenyl ether & $\mu g / L$ & 50 & 100 & EPA Method 8270 \\
\hline 2-sec-Butyl-4,6-dinitrophenol & $\mu g / L$ & 3.5 & 7 & Proposed DWS (EPA, 1990 ) \\
\hline Butylbenzyl phihalatt & & N. flag & No flag & Set by EPD/EMS \\
\hline Cadmium & $\mu g / L$ & 2.5 & 5 & Final DWS (CFR, 1991a) \\
\hline Calcium & & No $f \rightarrow g$ & No flag & Set by EPD/EMS \\
\hline Carbon disulfide & $\mu g / L$ & 5 & 10 & EPA Method 8240 \\
\hline Carbon tetrachloride & $\mu g / L$ & 2.5 & 5 & Final DWS (CFR, 1991a) \\
\hline Carbon-14 & $\mathrm{pCi} / \mathrm{L}$ & $1 E+03$ & $2 E+03$ & Final DWS (EPA, 1977) \\
\hline Carbonate & $\mu g / L$ & 500 & 1,000 & EPA Method 310.1 \\
\hline Cerium-141 & $\mathrm{pCi} / \mathrm{L}$ & $1.5 E+02$ & $3 E+02$ & Final DWS (EPA, 1977) \\
\hline Cerium-144 & $\mathrm{pCi} / \mathrm{L}$ & $1.31 E+02$ & $2.61 E+02$ & Proposed DWS (EPA, 1991) \\
\hline Cesium-134 & $\mathrm{pCi} / \mathrm{L}$ & $4.07 E+01$ & $8.13 E+01$ & Proposed DWS (EPA, 1991) \\
\hline Cesium-137 & $\mathrm{pCi} / \mathrm{L}$ & $1 E+02$ & $2 E+02$ & Final DWS (EPA, 1977) \\
\hline Chlordane & $\mu g / L$ & 1 & 2 & Final DWS (CFR, 1991a) \\
\hline Chloride & $\mu g / L$ & 125,000 & 250,000 & Secondary DWS (CFR, $1991 \mathrm{~b}$ ) \\
\hline 4-Chloroaniline & , و & 50 & 100 & EPA Method 8270 \\
\hline Chlorobenzene & $\mu g / L$ & 5 & 10 & EPA Method 8240 \\
\hline Chlorobenzilate & $\mu g / L$ & 50 & 100 & EPA Method 8270 \\
\hline Criloroethane & $\mu g / L$ & 5 & 10 & EPA Method 8240 \\
\hline Chloroethene (Vinyl chloride) & $\mu g / L$ & 1 & 2 & Final DWS (CFR, 1991a) \\
\hline Chloroethyl vinyl ether & $\mu g / L$ & 5 & 10 & EPA Method 8240 \\
\hline 2-Chloroethyi vinyl ether & $\mu g / L$ & 5 & 10 & EPA Method 8240 \\
\hline Chloroform & $\mu g / L$ & 50 & 100 & Final DWS (CFR, 1991a) \\
\hline para-Chloro-meta-cresol & $\mu \mathrm{g} / \mathrm{L}$ & 50 & 100 & SFA Method 8270 \\
\hline Chloromethane (Methyl chloride) & $\mu g / L$ & 5 & 10 & EPA Method 8240 \\
\hline 2-Chloronaphthalene & $\mu \mathrm{g} / \mathrm{L}$ & 50 & 100 & EPA Method 8240 \\
\hline 2-Chlorophenol & $\mu g / L$ & 50 & 100 & EPA Method 8270 \\
\hline
\end{tabular}




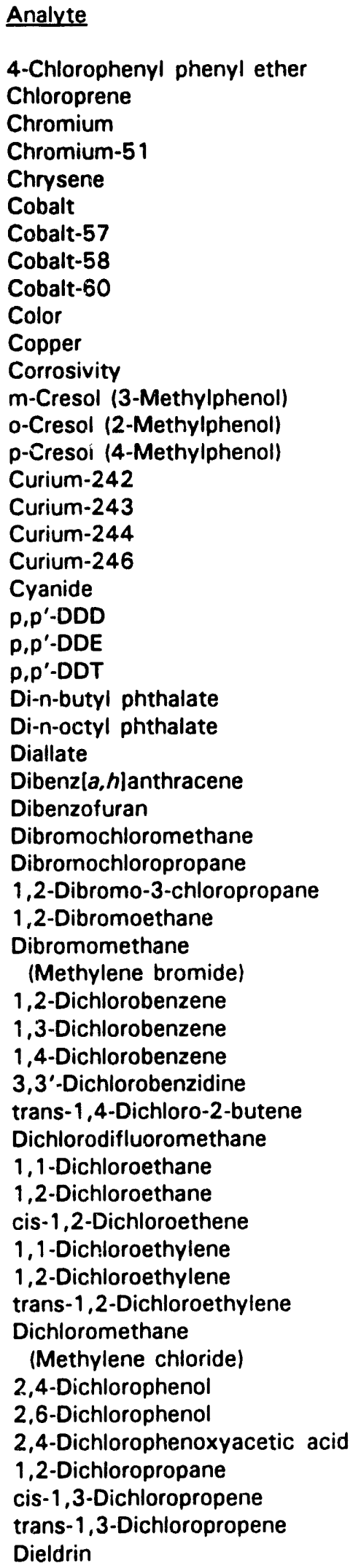

\begin{tabular}{|c|c|c|c|}
\hline Unit & Flag 1 & Flag 2 & Source \\
\hline$\mu g / L$ & 50 & 100 & EPA Method 8270 \\
\hline$\mu g / L$ & 1,000 & 2,000 & EPA Method 8240 \\
\hline$\mu g / L$ & 50 & 100 & Final DWS (CFR, 1991a) \\
\hline $\mathrm{pCi} / \mathrm{L}$ & $3 E+03$ & $6 E+03$ & Final DWS (EPA, 1977) \\
\hline$\mu g / L$ & 0.1 & 0.2 & Proposed DWS (EPA, 1990) \\
\hline$\mu g / L$ & 20 & 40 & EPA Method 6010 \\
\hline $\mathrm{pCi} / \mathrm{L}$ & $5 E+02$ & $1 E+03$ & Final DWS (EPA, 1977) \\
\hline $\mathrm{pCi} / \mathrm{L}$ & $4.5 E+03$ & $9 E+03$ & Final DWS (EPA, 1977) \\
\hline $\mathrm{pCi} / \mathrm{L}$ & $\begin{array}{l}5 E+01 \\
\text { No flag }\end{array}$ & $\begin{array}{l}1 E+02 \\
\text { No flag }\end{array}$ & $\begin{array}{l}\text { Final DWS (EPA, 1977) } \\
\text { Set by EPD/EMS }\end{array}$ \\
\hline$\mu g / L$ & 650 & 1,300 & Final DWS (CFR, 1991a) \\
\hline & No flag & No flag & Set by EPD/EMS \\
\hline$\mu g / L$ & 50 & 100 & EPA Method 8270 \\
\hline$\mu g / L$ & 50 & 100 & EPA Method 8270 \\
\hline$\mu g / L$ & 50 & 100 & EPA Method 8270 \\
\hline $\mathrm{pCi} / \mathrm{L}$ & $6.65 E+01$ & $1.33 E+02$ & Proposed DWS (EPA, 1991) \\
\hline $\mathrm{pCi} / \mathrm{L}$ & $4.15 E+00$ & $8.3 E+00$ & Proposed DWS (EPA, 1991) \\
\hline $\mathrm{pCi} / \mathrm{L}$ & $4.92 E+00$ & $9.84 E+00$ & Proposed DWS (EPA, 1991) \\
\hline $\mathrm{pCi} / \mathrm{L}$ & $3.14 E+00$ & $6.27 E+00$ & Proposed DWS (EPA, 1991) \\
\hline$\mu \mathrm{g} / \mathrm{L}$ & 100 & 200 & Proposed DWS (EPA, 1990) \\
\hline$\mu g / L$ & 2.5 & 5 & EPA Method 8080 \\
\hline$\mu g / L$ & 2.5 & 5 & EPA Method 8080 \\
\hline$\mu g / L$ & 2.5 & 5 & EPA Method 8080 \\
\hline & No flag & No flag & Set by EPD/EMS \\
\hline & No flag & No flag & Set by EPD/EMS \\
\hline$\mu g / L$ & 50 & 100 & EPA Method 8270 \\
\hline$\mu g / L$ & 0.15 & 0.3 & Proposed DWS (EPA, 1990) \\
\hline$\mu g / L$ & 50 & 100 & EPA Method 8270 \\
\hline$\mu g / L$ & 50 & 100 & Final DWS (CFR, 1991a) \\
\hline$\mu g / L$ & 0.1 & 0.2 & Final DWS (CFR, 1991a) \\
\hline$\mu g / L$ & 250 & 500 & EPA Method 8240 \\
\hline$\mu \mathrm{g} / \mathrm{L}$ & 100 & 200 & EPA Method 8240 \\
\hline$\mu g / L$ & 5 & 10 & EPA Method 8240 \\
\hline$\mu \mathrm{g} / \mathrm{L}$ & 300 & 600 & rinal DWS (CFR, 1991a) \\
\hline$\mu g / L$ & 50 & 100 & EPA Method 8270 \\
\hline$\mu g / L$ & 37.5 & 75 & Final DWS (CFR, 1991a) \\
\hline$\mu g / L$ & 50 & 100 & EPA Method 8270 \\
\hline$\mu g / L$ & 150 & 300 & EPA Method 8240 \\
\hline$\mu g / L$ & 5 & 10 & EPA Method 8240 \\
\hline$\mu g / L$ & 5 & 10 & EPA Method 8240 \\
\hline$\mu \mathrm{g} / \mathrm{L}$ & 2.5 & 5 & Final DWS (CFR, 1991a) \\
\hline$\mu g / L$ & 35 & 70 & Final DWS (CFR, 1991a) \\
\hline$\mu g / L$ & 3.5 & 7 & Final DWS (CFR, 1991a) \\
\hline$\mu g / L$ & 25 & 50 & EPA Method 8240 \\
\hline$\mu g / L$ & 50 & 100 & Final DWS (CFR, 1991a) \\
\hline & No flag & No flag & Set by EPD/EMS \\
\hline$\mu g / L$ & 50 & 100 & EPA Method 8270 \\
\hline$\mu g / L$ & 50 & 100 & EPA Method 8270 \\
\hline$\mu g / L$ & 35 & 70 & Final DWS (CFR, 1991a) \\
\hline$\mu g / L$ & 2.5 & 5 & Final DWS !CFR, 1991a) \\
\hline$\mu g / L$ & 5 & 10 & EPA Method 8240 \\
\hline$\mu g / L$ & 5 & 10 & EPA Method 8240 \\
\hline$\mu \mathrm{g} / \mathrm{L}$ & 2.5 & 5 & EPA Method 8080 \\
\hline
\end{tabular}




\begin{tabular}{|c|c|c|c|c|}
\hline Analyte & Unit & Flag 1 & Flag 2 & $\underline{\text { Source }}$ \\
\hline Diethyl phthalate & & No flag & No flag & Set by EPD/EMS \\
\hline Dimethoate & $\mu g / L$ & 50 & 100 & EPA Method 8270 \\
\hline p-Dimethylaminoazobenzene & $\mu g / L$ & 50 & 100 & EPA Method 8270 \\
\hline p-(Dimethylamino)ethylbenzene & $\mu g / L$ & 50 & 100 & EPA Method 8270 \\
\hline 7,12-Dimethylbenz[a]anthracene & $\mu g / L$ & 50 & 100 & EPA Method 8270 \\
\hline 3,3'-Dimethylbenzidine & $\mu g / L$ & 50 & 100 & EPA Method 8270 \\
\hline a,a-Dimethylphenethylamine & $\mu g / L$ & 50 & 100 & EPA Method 8270 \\
\hline 2,4-Dimethyl phenol & $\mu g / L$ & 50 & 100 & EPA Method 8270 \\
\hline Dimethyl phthalate & & No flag & No flag & Set by EPD/EMS \\
\hline 1,3-Dinitrobenzene & $\mu g / L$ & 50 & 100 & EPA Method 8270 \\
\hline 4,6-Dinitro-ortho-cresol & $\mu g / L$ & 250 & 500 & EPA Method 8270 \\
\hline 2,4-Dinitrophenol & $\mu g / L$ & 250 & 500 & EPA Method 8270 \\
\hline 2,4-Dinitrotoluene & $\mu g / L$ & 50 & 100 & EPA Method 8270 \\
\hline 2,6-Dinitrotoluene & $\mu g / L$ & 50 & 100 & EPA Method 8270 \\
\hline 1,4-Dioxane & $\mu g / L$ & 50 & 100 & EPA Method 8270 \\
\hline Diphenylamine & $\mu g / L$ & 50 & 100 & EPA Method 8270 \\
\hline 1,2-Diphenylhydrazine & $\mu g / L$ & 50 & 100 & EPA Method 8270 \\
\hline Dissolved organic carbon & $\mu g / L$ & 5,000 & 10,000 & EPA Method 9060 \\
\hline Disulfoton & $\mu g / L$ & 50 & 100 & EPA Method 8270 \\
\hline Eh & & No flag & No flag & Set by EPD/EMS \\
\hline alpha-Endosulfan & $\mu g / L$ & 50 & 100 & EPA Method 8270 \\
\hline beta-Endosulfan & $\mu \mathrm{g} / \mathrm{L}$ & 50 & 100 & EPA Method 8270 \\
\hline Endosulfan I & $\mu g / L$ & 2.5 & 5 & EPA Method 8080 \\
\hline Endosulfan II & $\mu g / L$ & 2.5 & 5 & EPA Method 8080 \\
\hline Endosulfan sulfate & $\mu g / L$ & 2.5 & 5 & EPA Method 8080 \\
\hline Endrin & $\mu g / L$ & 0.1 & 0.2 & Final DWS (CFR, 1991a) \\
\hline Endrin aldehyde & $\mu g / L$ & 2.5 & 5 & EPA Method 8080 \\
\hline Endrin ketone & & No flag & No flag & Set by EPD/EMS \\
\hline Ethyl methacrylate & $\mu g / L$ & 50 & 100 & EPA Method 8270 \\
\hline Ethyl methanesulfonate & $\mu \mathrm{g} / \mathrm{L}$ & 50 & 100 & EPA Method 8270 \\
\hline Ethylbenzene & $\mu g / L$ & 350 & 700 & Final DWS (CFR, 1991a) \\
\hline Europium-154 & $\mathrm{pCi} / \mathrm{L}$ & $1 E+02$ & $2 E+02$ & Final DWS (EPA, 1977) \\
\hline Europium-155 & $\mathrm{pCi} / \mathrm{L}$ & $3 E+02$ & $6 E+02$ & Final DWS (EPA, 1977) \\
\hline Famphur & $\mu g / L$ & 50 & 100 & EPA Method 8270 \\
\hline Fluoranthene & $\mu \mathrm{g} / \mathrm{L}$ & 50 & 100 & EPA Method 8270 \\
\hline Fluorene & $\mu g / L$ & 50 & 100 & EPA Method 8270 \\
\hline Fluoride & $\mu \mathrm{g} / \mathrm{L}$ & 2,000 & 4,000 & Final DWS (CFR, 1991a) \\
\hline Gross alpha & $\mathrm{pCi} / \mathrm{L}$ & $7.5 E+00$ & $1.5 E+01$ & Final DWS (CFR, 1991a) \\
\hline Heptachlor & $\mu g / L$ & 0.2 & 0.4 & Final DWS (CFR, 1991a) \\
\hline Heptachlor epoxide & $\mu g / L$ & 0.1 & 0.2 & Final DWS (CFR, 1991a) \\
\hline Heptachlorodibenzo-p-dioxin & & & & \\
\hline isomers & $\mu g / L$ & 0.00325 & 0.0065 & EPA Method 8280 \\
\hline $1,2,3,4,6,7,8$-Heptachlorodibenzo- & & & & \\
\hline p-dioxin & $\mu g / L$ & 0.00325 & 0.0065 & EPA Method 8280 \\
\hline Heptachlorodibenzo-p-furan & & & & \\
\hline isomers & $\mu g / L$ & 0.00225 & 0.0045 & EPA Method 8280 \\
\hline $\begin{array}{l}1,2,3,4,6,7,8 \text {-Heptachlorodibenzo- } \\
\text { p-furan }\end{array}$ & $\mu g / L$ & 0.00225 & 0.0045 & EPA Method 8280 \\
\hline Hexachlorobenzene & $\mu g / L$ & 0.5 & 1 & Proposed DWS (EPA, 1990) \\
\hline Hexachlorobutadiene & $\mu g / L$ & 50 & 100 & EPA Method 8270 \\
\hline Hexachlorocyclopentadiene & $\mu g / L$ & 25 & 50 & Proposed DWS (EPA, 1990) \\
\hline $\begin{array}{l}\text { Hexachlorodibenzo-p-dioxin isomers } \\
1,2,3,4,7,8 \text {-Hexachlorodibenzo- }\end{array}$ & $\mu g / L$ & 0.00225 & 0.0045 & EPA Method 8280 \\
\hline p-dioxin & $\mu g / L$ & 0.00225 & 0.0045 & EPA Method 8280 \\
\hline Hexachlorodibenzo-p-furan isomers & $\mu g / L$ & 0.002 & 0.004 & EPA Method 8280 \\
\hline
\end{tabular}


Analyte

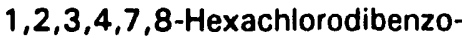
p-furan

Hexachloroethane

Hexachlorophene

Hexachloropropene

2-Hexanone

Indenol 1,2,3-c, olpyrerie

lodine

lodine-129

lodine-131

lodomethane (Methyl iodide)

Iron

Iron-55

Iron-59

Isobutyl alcohol

Isodrin

Isophorone

Isosafrole

Kepone

Lanthanum-140

Lead

Lindane

Lithium

Magnesium

Manganese

Manganese-54

Mercury

Methacrylonitrile

Methapyrilene

Methoxychlor

3-Methylcholanthrene

2-Methyl-4,6-dinitrophenol

Methyl ethyl ketone

Methyl isobutyl ketone

Methyl methacrylate

Methyl methanesulfonate

2-Methylnaphthalene

Molybdenum

Naphthalene

1,4-Naphthoquinone

1-Naphthylamine

2-Naphthylamine

Neptunium-237

Nickel

Nickel-59

Nickel-63

Niobium-95

Nitrate as nitrogen

Nitrate-nitrite as nitrogen

Nitrite as nitrogen

2-Nitroaniline

3-Nitroaniline

4-Nitroaniline

Nitrobenzene

Nitrogen by Kjeldahl method
Unit

Flag 1

Flag 2

$\underline{\text { Source }}$

\begin{tabular}{|c|c|c|}
\hline $\begin{array}{l}\mu g / L \\
\mu g / L\end{array}$ & $\begin{array}{l}0.002 \\
50\end{array}$ & $\begin{array}{l}0.004 \\
100\end{array}$ \\
\hline$\mu g / L$ & 250 & 500 \\
\hline$\mu g / L$ & 50 & 100 \\
\hline$\mu g / L$ & 100 & 200 \\
\hline$\mu g / L$ & 50 & 100 \\
\hline$\mu g / L$ & 500 & 1,000 \\
\hline $\mathrm{pCi} / \mathrm{L}$ & 5E-01 & $1 E+00$ \\
\hline $\mathrm{pCi} / \mathrm{L}$ & $1.5 E+\infty 0$ & $3 E+00$ \\
\hline$\mu g / L$ & 75 & 150 \\
\hline$\mu g / L$ & 150 & 300 \\
\hline $\mathrm{pCi} / \mathrm{L}$ & $1 E+03$ & $2 E+03$ \\
\hline pCi/L & $1 E+02$ & $2 E+02$ \\
\hline$\mu \mathrm{g} / \mathrm{L}$ & 500 & 1,000 \\
\hline$\mu g / L$ & 50 & 100 \\
\hline$\mu g / L$ & 50 & 100 \\
\hline$\mu g / L$ & 50 & 100 \\
\hline$\mu g / L$ & 50 & 100 \\
\hline $\mathrm{pCi} / \mathrm{L}$ & $3 E+01$ & $6 E+01$ \\
\hline$\mu g / L$ & 7.5 & 15 \\
\hline$\mu g / L$ & 0.1 & 0.2 \\
\hline$\mu g / L$ & 25 & 50 \\
\hline & No flag & $\begin{array}{l}\text { No flag } \\
50\end{array}$ \\
\hline$\mu \mathrm{g} / \mathrm{L}$ & 25 & $\begin{array}{l}50 \\
3 E+02\end{array}$ \\
\hline pCi/L & $\begin{array}{l}1.5 E+02 \\
1\end{array}$ & $\begin{array}{l}3 E+02 \\
2\end{array}$ \\
\hline $\begin{array}{l}\mu g / L \\
\mu g / L\end{array}$ & 250 & 500 \\
\hline$\mu g / L$ & 50 & 100 \\
\hline$\mu g / L$ & 20 & 40 \\
\hline$\mu g / L$ & 50 & 100 \\
\hline$\mu g / L$ & 250 & 500 \\
\hline & No flag & No flag \\
\hline & No flag & No flag \\
\hline$\mu \mathrm{g} / \mathrm{L}$ & 50 & 100 \\
\hline$\mu \mathrm{g} / \mathrm{L}$ & 50 & 100 \\
\hline$\mu g / L$ & 50 & 100 \\
\hline$\mu \mathrm{g} / \mathrm{L}$ & 250 & 500 \\
\hline$\mu g / L$ & 50 & 100 \\
\hline$\mu g / L$ & 50 & 100 \\
\hline$\mu g / L$ & 50 & 100 \\
\hline$\mu \mathrm{g} / \mathrm{L}$ & 50 & 100 \\
\hline $\mathrm{pCi} / \mathrm{L}$ & $3.53 E+00$ & $7.06 E+00$ \\
\hline$\mu \mathrm{g} / \mathrm{L}$ & 50 & 100 \\
\hline $\mathrm{pCi} / \mathrm{L}$ & $1.5 E+02$ & $3 E+02$ \\
\hline $\mathrm{pCi} / \mathrm{L}$ & $2.5 E+01$ & $5 E+01$ \\
\hline $\mathrm{pCi} / \mathrm{L}$ & $1.5 E+02$ & $3 E+02$ \\
\hline$\mu \mathrm{g} / \mathrm{L}$ & 5,000 & 10,000 \\
\hline$\mu g / L$ & 5,000 & 10,000 \\
\hline$\mu \mathrm{g} / \mathrm{L}$ & 500 & 1,000 \\
\hline$\mu g / L$ & 50 & 100 \\
\hline$\mu g / L$ & 50 & 100 \\
\hline$\mu g / L$ & 50 & 100 \\
\hline$\mu g / L$ & 50 & 100 \\
\hline$\mu g / L$ & 500 & 1,000 \\
\hline
\end{tabular}

EPA Method 8280

EPA Method 8270

EPA Method 8270

EPA Method 8270

EPA Method 8240

EPA Method 8270

EPA Method 415

Final DWS (EPA, 1977)

Final DWS (EPA, 1977)

EPA Method 8240

Secondary DWS (CFR, 1991b)

Final DWS (EPA, 1977)

Final DWS (EPA, 1977)

EPA Method 8240

EPA Method 8270

EPA Method 8270

EPA Method 8270

EPA Method 8270

Final DWS (EPA, 1977)

Final DWS (CFR, 1991a)

Final DWS (CFR, 1991a)

EPA Method 6010

Set by EPD/EMS

Secondary DWS (CFR, 1991b)

Final DWS (EPA, 1977)

Final DWS (CFR, 1991a)

EPA Method 8240

EPA Method 8270

Final DWS (CFR, 1991a)

EPA Method 8270

EPA Method 8270

Set by EPD/EMS

Set by EPD/EMS

EPA Method 8270

EPA Method 8270

EPA Method 8270

EPA Method 6010

EPA Method 8270

EPA Method 8270

EPA Method 8270

EPA Method 8270

Proposed DWS (EPA, 1991)

Proposed DWS (EPA, 1990$)$

Final DWS (EPA, 1977)

Final DWS (EPA, 1977)

Final DWS (EPA, 1977)

Final DWS (CFR, 1991a)

Final DWS (CFR, 1991a)

Final DWS (CFR, 1991a)

EPA Method 8270

EPA Method 8270

EPA Method 8270

EPA Method 8270

EPA Method 351.2 


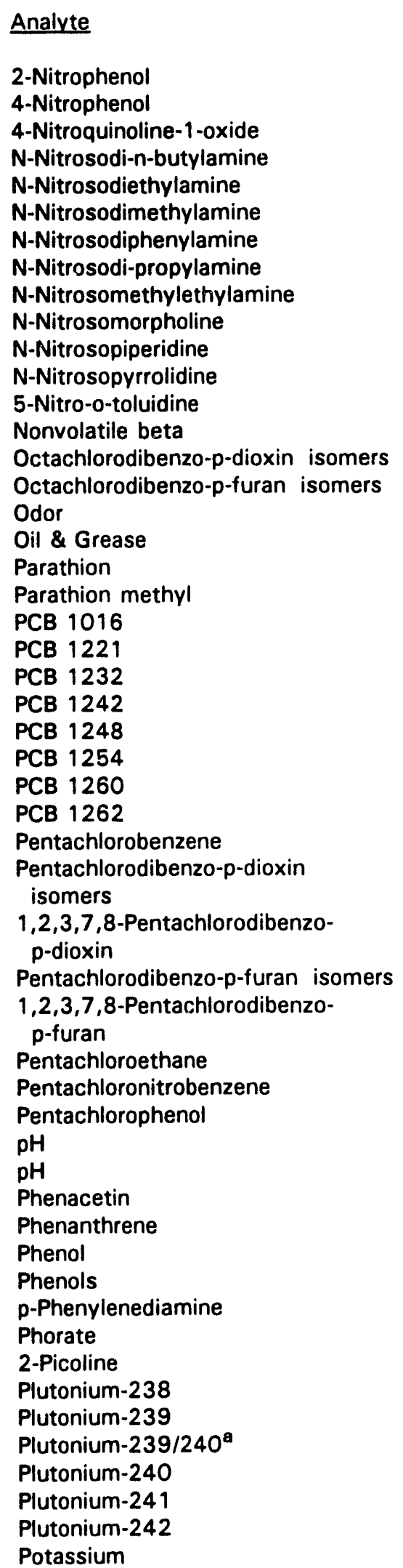

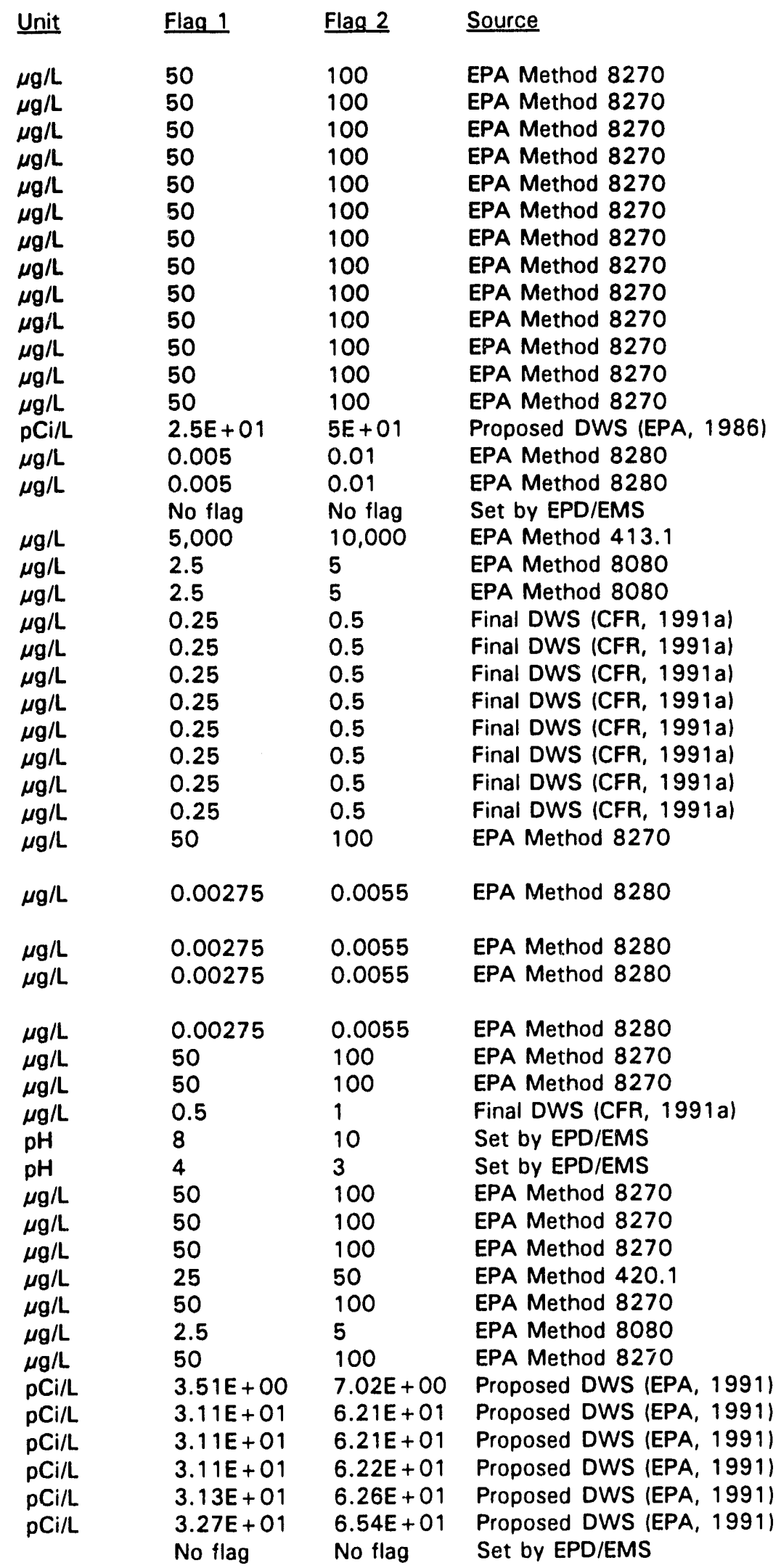




\begin{tabular}{|c|c|c|c|c|}
\hline Analyte & Unit & Flag 1 & Flag 2 & Source \\
\hline Potassium-40 & $\mathrm{pCi} / \mathrm{L}$ & $1.5 E+02$ & $3 E+02$ & Proposed DWS (EPA, 1986) \\
\hline Pronamid & $\mu g / L$ & 50 & 100 & EPA Method 8270 \\
\hline Propionitrile & $\mu g / L$ & 1,000 & 2,000 & EPA Method 8240 \\
\hline Pyrene & $\mu g / L$ & 50 & 100 & EPA Method 8270 \\
\hline Pyridine & $\mu g / L$ & 50 & 100 & EPA Method 8270 \\
\hline Radium-226 & $\mathrm{pCi} / \mathrm{L}$ & $7.85 E+00$ & $1.57 E+01$ & Proposed DWS (EPA, 1991) \\
\hline Radium-228 & $\mathrm{pCi} / \mathrm{L}$ & $3.93 E+00$ & $7.85 E+00$ & Proposed DWS (EPA, 1991) \\
\hline Radon-222 & $\mathrm{pCi} / \mathrm{L}$ & $1.5 E+02$ & $3 E+02$ & Proposed DWS (EPA, 1991) \\
\hline Ruthenium-103 & $\mathrm{pCi} / \mathrm{L}$ & $1 E+02$ & $2 E+02$ & Final DWS (EPA, 1977) \\
\hline Ruthenium-106 & $\mathrm{pCi} / \mathrm{L}$ & $1.5 E+01$ & $3 E+01$ & Final DWS (EPA, 1977) \\
\hline Safrole & $\mu g / L$ & 50 & 100 & EPA Method 8270 \\
\hline Selenium & $\mu g / L$ & 25 & 50 & Final DWS (CFR, 1991a) \\
\hline Silica & & No flag & No flag & Set by EPD/EMS \\
\hline Silver & $\mu g / L$ & 25 & 50 & Final DWS (CFR, 1991a) \\
\hline Sodium & & No flag & No flag & Set by EPD/EMS \\
\hline Sodium-22 & $\mathrm{pCi} / \mathrm{L}$ & $2.33 E+02$ & $4.66 \mathrm{E}+02$ & Proposed DWS (EPA, 1991) \\
\hline Specific conductance & $\mu \mathrm{S} / \mathrm{cm}$ & 250 & 500 & Set by EPD/EMS \\
\hline Strontium-89 & $\mathrm{pCi} / \mathrm{L}$ & $1 E+01$ & $2 E+01$ & Final DWS (EPA, 1977) \\
\hline Strontium-89/90 & $\mathrm{pCi} / \mathrm{L}$ & $4 E+00$ & $8 E+00$ & Final DWS (CFR, 1991a) \\
\hline Strontium-90 & $\mathrm{pCi} / \mathrm{L}$ & $4 E+00$ & $8 E+00$ & Final DWS (CFR, 1991a) \\
\hline Styrene & $\mu g / L$ & 50 & 100 & Final DWS (CFR, 1991a) \\
\hline Sulfate & $\mu g / L$ & 200,000 & 400,000 & Proposed DWS (EPA, 1990) \\
\hline Sulfide & $\mu g / L$ & 5,000 & 10,000 & EPA Method 9030 \\
\hline Sulfotepp & $\mu g / L$ & 50 & 100 & EPA Method 8270 \\
\hline Surfactants & & No flag & No flag & Set by EPD/EMS \\
\hline $2,3,7,8-T C D D$ & $\mu g / L$ & 0.00225 & 0.0045 & EPA Method 8280 \\
\hline $2,3,7,8-T C D F$ & $\mu g / L$ & 0.002 & 0.004 & EPA Method 8280 \\
\hline Technetium-99 & $\mathrm{pCi} / \mathrm{L}$ & $4.5 E+02$ & $9 E+02$ & Final DWS (EPA, 1977) \\
\hline $\begin{array}{l}\text { 1,2,4,5-Tetrachlorobenzene } \\
\text { Tetrachlorodibenzo-p-dioxin }\end{array}$ & $\mu g / L$ & 50 & 100 & EPA Method 8270 \\
\hline isomers & $\mu g / L$ & 0.00225 & 0.0045 & EPA Method 8280 \\
\hline Tetrachlorodibenzo-p-furan & & & & \\
\hline isomers & $\mu g / L$ & 0.002 & 0.004 & EPA Method 8280 \\
\hline $1,1,1,2$-Tetrachloroethane & $\mu g / L$ & 5 & 10 & EPA Method 8240 \\
\hline $1,1,2,2$-Tetrachloroethane & $\mu g / L$ & 5 & 10 & rPA Method 8240 \\
\hline Tetrachloroethylene & $\mu g / L$ & 2.5 & 5 & Final DWS (CFR, 1991a) \\
\hline 2,3,4,6-Tetrachlorophenol & $\mu g / L$ & 50 & 100 & EPA Method 8270 \\
\hline Tetraethyl dithiopyrophosphate & $\mu g / L$ & 50 & 100 & EPA Method 8270 \\
\hline Thallium & $\mu g / L$ & 0.5 & 1 & Proposed DWS (EPA, 1990) \\
\hline Thionazin & $\mu g / L$ & 50 & 100 & EPA Method 8270 \\
\hline Thorium-228 & $\mathrm{pCi} / \mathrm{L}$ & $6.25 E+01$ & $1.25 E+02$ & Proposed DWS (EPA, 1991) \\
\hline Thorium-230 & $\mathrm{pCi} / \mathrm{L}$ & $3.96 \mathrm{E}+01$ & $7.92 E+01$ & Proposed DWS (EPA, 1991) \\
\hline Thorium-232 & $\mathrm{pCi} / \mathrm{L}$ & $4.4 E+01$ & $8.8 E+01$ & Proposed DWS (EPA, 1991) \\
\hline Thorium-234 & $\mathrm{pCi} / \mathrm{L}$ & $2 E+02$ & $4.01 E+02$ & Proposed DWS (EPA, 1991) \\
\hline Tin & $\mu g / L$ & 10 & 20 & EPA Method 282.2 \\
\hline Tin-113 & $\mathrm{pCi} / \mathrm{L}$ & $1.5 E+02$ & $3 E+02$ & Final DWS (EPA, 1977) \\
\hline Toluene & $\mu g / L$ & 500 & 1,000 & Final DWS (CFR, 1991a) \\
\hline o-Toluidine & $\mu g / L$ & 50 & 100 & EPA Method 8270 \\
\hline Total carbon & $\mu g / L$ & 5,000 & 10,000 & EPA Method 9060 \\
\hline Total dissolved solids & & No flag & No flag & Set by EPD/EMS \\
\hline Total hydrocarbons & $\mu g / L$ & 5,000 & 10,000 & EPA Method 418.1 \\
\hline Total inorganic carbon & $\mu g / L$ & 5,000 & 10,000 & EPA Method 9060 \\
\hline Total organic carbon & $\mu g / L$ & 5,000 & 10,000 & EPA Method 9060 \\
\hline Total organic halogens & $\mu g / L$ & 25 & 50 & EPA Method 9020 \\
\hline Total organic nitrogen & $\mu g / L$ & 500 & 1,000 & EPA Method 420 \\
\hline
\end{tabular}


Analyte

Total petroleum hydrocarbons

Total phosphates (as P)

Total phosphorus

Total radium

Total silica

Total trihalomethanes

Toxaphene

2,4,5-TP (Silvex)

Tributyl phosphate

1,2,4-Trichlorobenzene

1,1,1-Trichloroethane

1,1,2-Trichloroethane

Trichloroethylene

Trichlorofluoromethane

2,4,5-Trichlorophenol

2,4,6-Trichlorophenol

2,4,5-Trichlorophenoxyacetic acid

1,2,3-Trichloropropane

$0,0,0$-Triethyl phosphorothioate

1,3,5-Trinitrobenzene

Tritium

Turbidity

Uranium

Uranium alpha activity

Uranium-233/234

Uranium-234

Uranium-235

Uranium-238

Vanadium

Vinyl acetate

Xylenes

Zinc

Zinc-65

Zirconium-95

Zirconium/Niobium-95 ${ }^{\mathrm{a}}$

\begin{tabular}{|c|c|c|}
\hline Unit & Flag 1 & Flag 2 \\
\hline$\mu g / L$ & $\begin{array}{l}5,000 \\
\text { No flag }\end{array}$ & $\begin{array}{l}10,000 \\
\text { No flag }\end{array}$ \\
\hline $\mathrm{pCi} / \mathrm{L}$ & $\begin{array}{l}\text { No flag } \\
2.5 E+00\end{array}$ & $\begin{array}{l}\text { No flag } \\
5 E+00\end{array}$ \\
\hline$\mu g / L$ & 500 & 1,000 \\
\hline$\mu g / L$ & 50 & 100 \\
\hline$\mu g / L$ & 1.5 & 3 \\
\hline$\mu g / L$ & 25 & 50 \\
\hline$\mu g / L$ & 50 & 100 \\
\hline$\mu g / L$ & 4.5 & 9 \\
\hline$\mu g / L$ & 100 & 200 \\
\hline$\mu g / L$ & 2.5 & 5 \\
\hline$\mu g / L$ & 2.5 & 5 \\
\hline$\mu g / L$ & 5 & 10 \\
\hline$\mu g / L$ & 50 & 100 \\
\hline$\mu g / L$ & 50 & 100 \\
\hline$\mu g / L$ & 2.5 & 5 \\
\hline$\mu g / L$ & 5 & 10 \\
\hline$\mu g / L$ & 50 & 100 \\
\hline$\mu g / L$ & 50 & 100 \\
\hline $\mathrm{pCi} / \mathrm{mL}$ & $\begin{array}{l}1 E+01 \\
\text { No flag }\end{array}$ & $\begin{array}{l}2 E+01 \\
\text { No flag }\end{array}$ \\
\hline$\mu g / L$ & 10 & 20 \\
\hline $\mathrm{pCi} / \mathrm{L}$ & $1.5 E+01$ & $3 E+01$ \\
\hline$p C i / L$ & 6.9E-00 & $1.38 E+01$ \\
\hline $\mathrm{pCi} / \mathrm{L}$ & $6.95 E+00$ & $1.39 E+01$ \\
\hline $\mathrm{pCi} / \mathrm{L}$ & $7.25 E+00$ & $1.45 E+01$ \\
\hline $\mathrm{pCi} / \mathrm{L}$ & $7.3 E+00$ & $1.46 E+01$ \\
\hline$\mu g / L$ & 50 & 100 \\
\hline$\mu g / L$ & 5 & 10 \\
\hline$\mu g / L$ & 5,000 & 10,000 \\
\hline$\mu g / L$ & 2,500 & 5,000 \\
\hline $\mathrm{pCi} / \mathrm{L}$ & $1.5 E+02$ & $3 E+02$ \\
\hline $\mathrm{pCi} / \mathrm{L}$ & $1 E+02$ & $2 E+02$ \\
\hline $\mathrm{pCi} / \mathrm{L}$ & $1 E+02$ & $2 E+02$ \\
\hline
\end{tabular}

Source

EPA Method 418.1

Set by EPD/EMS

Set by EPD/EMS

Final DWS (CFR, 1991a)

EPA Method 6010

Final DWS (CFR, 1991a)

Final DWS (CFR, 1991a)

Final DWS (CFR, 1991a)

EPA Method 8270

Proposed DWS (EPA, 1990)

Final DWS (CFR, 1991a)

Proposed DWS (EPA, 1990)

Final DWS (CFR, 1991a)

EPA Method 8240

EPA Method 8270

EPA Method 8270

EPA Method 8150

EPA Method 8240

EPA Method 8270

EPA Method 8270

Final DWS (CFR, 1991a)

Set by EPD/EMS

Proposed DWS (EPA, 1991)

Proposed DWS (EPA, 1991)

Proposed DWS (EPA, 1991)

Proposed DWS (EPA, 1991)

Proposed DWS (EPA, 1991)

Proposed DWS (EPA, 1991)

EPA Method 6010

EPA Method 8240

Final DWS (CFR, 1991a)

Secondary DWS (CFR, 1991b)

Final DWS (EPA, 1977)

Final DWS (EPA, 1977)

Final DWS (EPA, 1977)

a For double radionuclide analyses where each separate radionuclide has its own standard, the more stringent standard is used.

\section{References}

APHA (American Public Health Association, American Water Works Association, and Water Pollution Control Federation), 1985. Standard Methods for the Examination of Water and Wastewater, 16th edition. Washington, DC.

CFR (Code of Federal Regulations), 1991a. National Primary Drinking Water Regulations, 40 CFR, Part 141, pp. 578-715. Washington, DC.

CFR (Code of Federal Regulations), 1991b. National Secondary Drinking Water Regulations, 40 CFR, Part 143, pp. 758-762. Washington, DC. 
EPA (U.S. Environmental Protection Agency), 1977. National Interim Primary Drinking Water Regulations, EPA-570/9-76-003. Washington, DC.

EPA (U.S. Environmental Protection Agency), 1986. Water Pollution Control; National Primary Drinking Water Regulations, Radionuclides (Proposed). Federal Register, September 30, 1986, pp. 34836-34862. Washington, DC.

EPA (U.S. Environmental Protection Agency). 1990. National Primary and Secondary Drinking Water Regulations; Synthetic Organic Chemicals and Inorganic Shemicals (Proposed Rule). Foderal Register, July 25, 1990, pp. 30369-30448. Washington, DC.

EPA (U.S. Environmental Protection Agency), 1991. National Primary Drinking Water Regulations; Radionuclides; Proposed Rule. Federal Register, July 18, 1991, pp. 33052-33127. Washington, DC. 


\section{Appendix C - Figures}




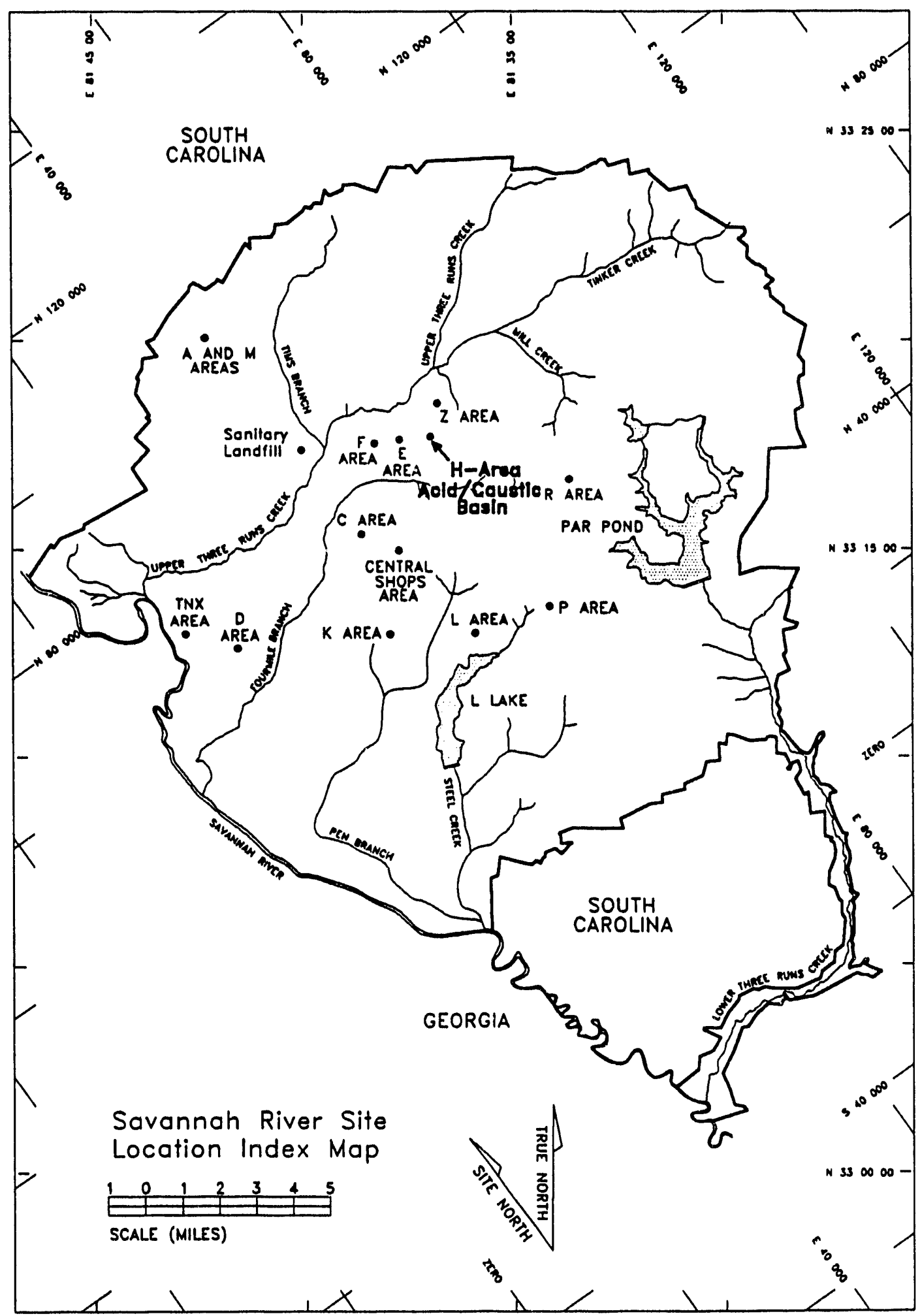

Figure 1. Location of the H-Area Acid/Caustic Basin at the Savannah River Site 


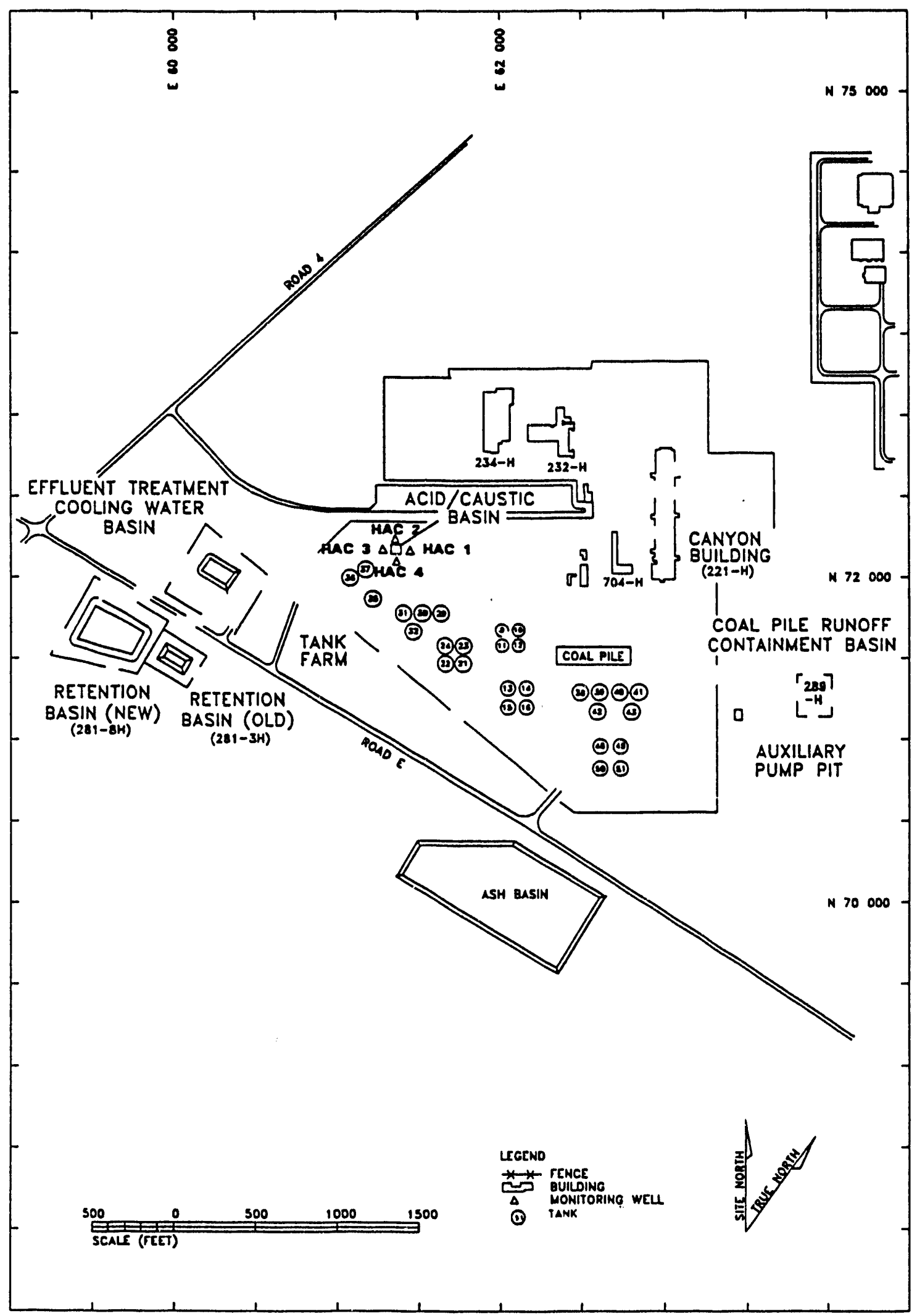

Figure 2. Location of Groundwater Monitoring Wells at the H-Area Acid/Caustic Basin 


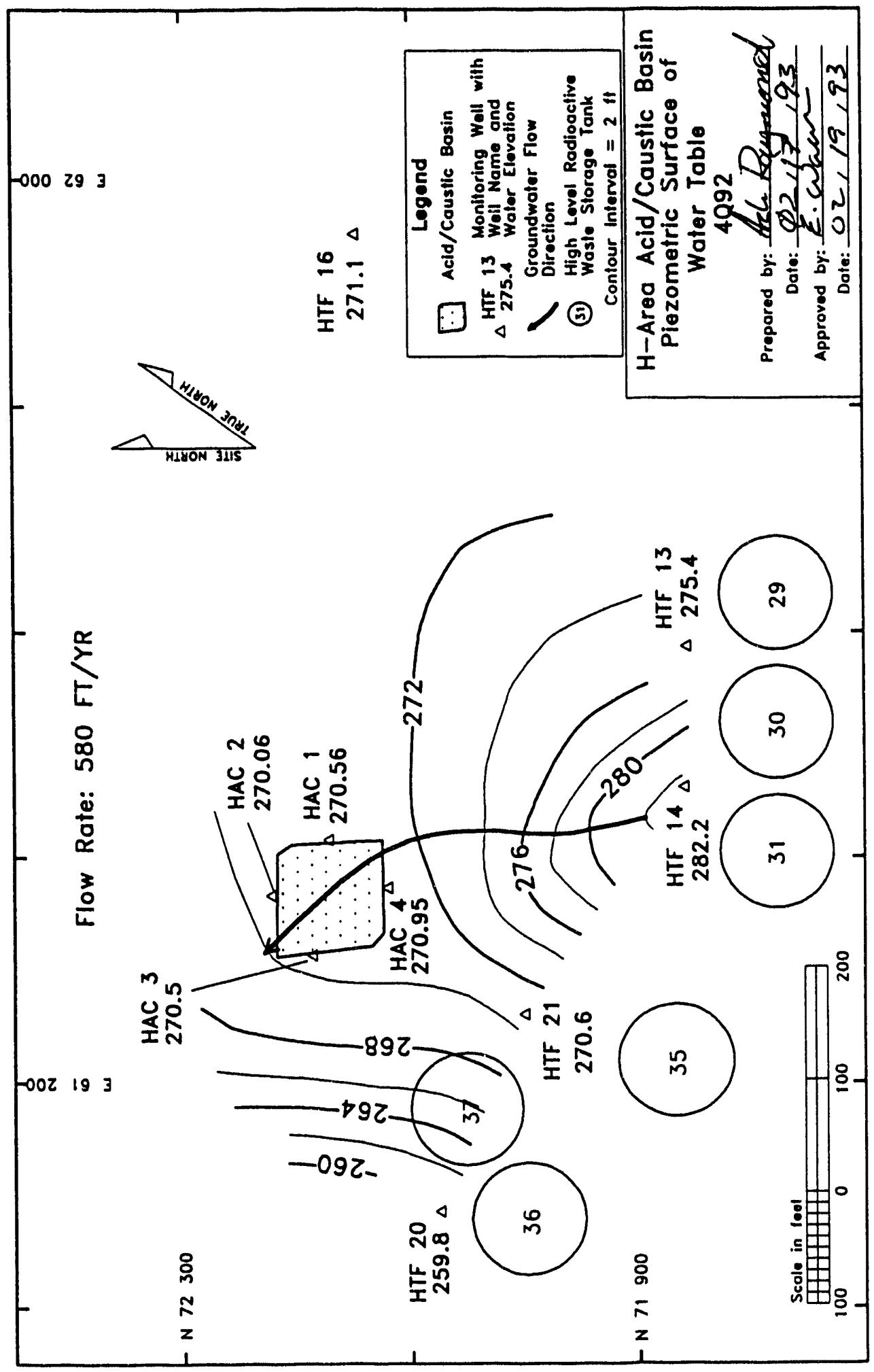




\section{Appendix D - Groundwater Monitoring Results Tables}




\section{Key to Reading the Tables}

The following abbreviations may appear in the tabular data:

B = sample collected from well using an open bucket bailer

$\mathrm{BA}=$ Barringer Laboratories, Inc.

$\mathrm{CN}=$ Clemson Technical Center, Inc.

$\mathrm{CS}=$ carbon steel

$\mathrm{D}=$ primary drinking water standard (PDWS)

$\mathrm{E}=$ exponential notation (e.g., $1.1 \mathrm{E}-09=1.1 \times 10^{-9}=0.0000000011$ )

EM = Environmental Protection Department/Environmental Monitoring Section (EPD/EMS)

Laboratory

$\mathrm{GE}=$ General Engineering Laboratories

GP = Environmental Physics, Inc.

$\mathrm{H}=$ holding time

1,2,3,4,6,7,8-HPCDD $=1,2,3,4,6,7,8$-heptachlorodibenzo-p-dioxin

$1,2,3,4,6,7,8$-HPCDF $=1,2,3,4,6,7,8$-heptachlorodibenzo-p-furan

$1,2,3,4,7,8$-HXCDD $=1,2,3,4,7,8$-hexachlorodibenzo-p-dioxin

$1,2,3,4,7,8$-HXCDF $=1,2,3,4,7,8$-hexachlorodibenzo-p-furan

Lindane = gamma-benzene hexachloride

$\mathrm{mg} / \mathrm{L}=$ milligrams per liter

Mod $=$ modifier

$\mathrm{msl}=$ mean sea level

MSL = million structures per liter

NTU $=$ turbidity unit

$\mathrm{P}=$ sample collected from well using a bladder pump

$\mathrm{PCB}=$ polychlorinated biphenyl

1,2,3,7,8-PCDD $=1,2,3,7,8$-pentachlorodibenzo-p-dioxin

1,2,3,7,8-PCDF $=1,2,3,7,8$-pentachlorodibenzo-p-furan

$\mathrm{pCi} / \mathrm{L}=$ picocuries per liter

$\mathrm{pCi} / \mathrm{mL}=$ picocuries per milliliter

PDWS = primary drinking water standard

$\mathrm{pH}=\mathrm{pH}$ unit

PVC = polyvinyl chloride

$\mathrm{S}=$ sample collected from well using a single-speed centrifugal downhole pump

Sp. conductance $=$ specific conductance

$\mathrm{SP}=$ Spencer Testing Services, Inc.

TCDD = tetrachlorodibenzo-p-dioxin

$\mathrm{TCDF}=$ tetrachlorodibenzo-p-furan

$\mathrm{TM}=\mathrm{TMA} /$ Eberline

$\mathrm{TOC}=$ top of casing

$\mathrm{V}$ = sample collected from well using a variable-speed pump

$\mathrm{WA}=$ Roy $\mathrm{F}$. Weston, Inc.

$\mu \mathrm{g} / \mathrm{L}=$ micrograms per liter

$\mu \mathrm{S} / \mathrm{cm}=$ microsiemens per centimeter 


\section{Holding Times}

Standard analytical methods include a limit, called holding time, on the maximum elapsed time between sample collection and extraction or analysis by the thoratory. In the data tables, a large dot $(\bullet)$ in the $\mathrm{H}$ (holding time) column indicates that holding time was exceeded. Analyses performed beyond holdin $\tilde{\imath}^{-}+$ime may not yield valid results.

The South Carolina Department of Health and Environmental Controi allows only 15 minutes to elapse between sampling and analysis for $\mathrm{pH}$. Thus, only field $\mathrm{pH}$ measurements can meet the holding time criterion; laboratory $\mathrm{pH}$ analyses will always exceed it.

Laboratory-initiated procedures for reducing the number of other analyses performed out of holding time include subcontractirg analyses when difficulties with equipment, personnel, or work load would prevent timely analyses. Beginning fourth quarter 1991, SRS reduced th. compensation to laboratories for analyses performed out of holding time.

\section{Data Qualification}

The contract laboratories continually assess tiseir own accuracy and precision according to U.S. Environmental Protection Agency (EPA) guidelines. They submit sample- or batchspecific quality assurance/quality control informarion either at the same time as analytical results or in a quarterly summary. Properly defined and used result modifiers (also referred to as qualifiers) can be a key component in assessing data useability. Result modifiers designed by EPD/EMS and provided to the primary laboratories are defined below. These modifiers appear in the data tables under the column "Mod."

Resuit modifier

(Blank)

$A^{a}$

$J^{\mathbf{a}}$

$L^{a}$

$M^{a}$

$\mathrm{R}^{\mathrm{a}}$

$T^{a}$

$v^{a}$

1

2

\section{Definition}

Data are not qualified. Number should be iriterpreted exactly as reported.

Value reported is the mean of two or more determinations.

Value is estimated because quantitation in the sample or in associated quality control samples did not meet specifications.

Value is off-scale high. The actual value is not known but is known to be greater than the value shown.

Presence of the analyte is verified but not quantified.

Result was rejected because performance requirements in the sample analysis or associated quality control analyses were not met.

Analyte was not detected; if present, it was below the criteria for detection.

Analyte was detected in the associated method blank.

Result may be an underestimation of the true value due to analytical bias.

Result may be an overestimation of the true value due to analytical bias. 


\section{Result modifier Definition}

3

6

a These codes are based on the STORET codes from EPA.
The associated result may be of poor precision (high variability) due to analytical bias.

The associated result is from a reanalysis performed out of holding time due to problems with an earlier analysis. 
Table 1. Constituents Exceeding Final Primary Drinking Water Standards for 1992

\begin{tabular}{|c|c|c|c|c|c|c|}
\hline Well & Constituent & Unit & 1092 & 2092 & 3092 & 4092 \\
\hline HAC & Tritium & $\mathrm{pCi} / \mathrm{mL}$ & $5.3 E+01$ & $3.9 E+01$ & $5.3 E+01$ & $5.1 E+01$ \\
\hline HAC & Tritium & $\mathrm{pCi} / \mathrm{mL}$ & $4.0 E+01$ & $5.0 E+01$ & $3.8 E+01$ & $4.2 E+01$ \\
\hline HAC & Tritium & $\mathrm{pCi} / \mathrm{mL}$ & $3.6 E+01$ & $3.6 E+01$ & $4.3 E+01$ & $4.4 E+01$ \\
\hline HAC & $\begin{array}{l}\text { Tritium } \\
\text { Tritium }\end{array}$ & $\begin{array}{l}\mathrm{pCi} / \mathrm{mL} \\
\mathrm{pCi} / \mathrm{mL}\end{array}$ & $\begin{array}{l}2.7 E+01 \\
N^{b}\end{array}$ & $\begin{array}{l}3.0 E+01 \\
\text { NA }\end{array}$ & $\begin{array}{l}4.0 E+01^{a} \\
4.1 E+01^{a}\end{array}$ & $\begin{array}{l}4.6 E+01 \\
N A\end{array}$ \\
\hline
\end{tabular}

a Duplicate/roplicate samples of tritium.

b $N A=$ not analyzed.

Table 2. Constituents Exceeding Half the Final Primary Drinking Water Standards, Other Flag 1 or Flag 2 Criteria, or the SRS Turbidity Standard

\begin{tabular}{|c|c|c|c|c|c|}
\hline Well & Constituent & Unit & 4092 & Mod & Flag \\
\hline HAC & $\begin{array}{l}\text { Iron } \\
\text { Lead }\end{array}$ & $\begin{array}{l}\mu g / L \\
\mu g / L\end{array}$ & $\begin{array}{l}716 \\
10\end{array}$ & & $\begin{array}{l}2 \\
1\end{array}$ \\
\hline HAC & $\begin{array}{l}\text { Iron } \\
\text { Specific conductence }\end{array}$ & $\begin{array}{l}\mu \mathrm{g} / \mathrm{L} \\
\mu \mathrm{S} / \mathrm{cm}\end{array}$ & $\begin{array}{l}2,070 \\
519\end{array}$ & $\begin{array}{l}V \\
J\end{array}$ & $\begin{array}{l}2 \\
2\end{array}$ \\
\hline HAC & $\begin{array}{l}\text { Iron } \\
\text { Lead } \\
\text { Manganese } \\
\text { Specific conductance }\end{array}$ & $\begin{array}{l}\mu \mathrm{g} / \mathrm{L} \\
\mu \mathrm{g} / \mathrm{L} \\
\mu \mathrm{g} / \mathrm{L} \\
\mu \mathrm{S} / \mathrm{cm}\end{array}$ & $\begin{array}{l}406 \\
13 \\
51 \\
266\end{array}$ & J & $\begin{array}{l}2 \\
1 \\
2 \\
1\end{array}$ \\
\hline HAC & Manganese & $\mu g / L$ & 29 & & 1 \\
\hline
\end{tabular}

Note: Constituents exceeding half the final PDWS appear italicized. These results do not include field data results. 
Table 3. Groundwater Monitoring Results for Individual Wells

WELL HAC 1

\begin{tabular}{|c|c|c|c|c|c|c|}
\hline SAS coord. & Lat/Longitude & Screen Zone Elevation & Top of Casing & Casing & Pump & Formation \\
\hline $\begin{array}{l}N 72171.0 \\
E 61415.2\end{array}$ & $\begin{array}{l}33.285599 \\
81.645272{ }^{\circ} \mathrm{W}\end{array}$ & 278.8-258.8 ft msl & $298.4 \mathrm{ft} \mathrm{msl}$ & 4" PVC & $\mathbf{s}$ & Water table \\
\hline \multicolumn{2}{|c|}{ SAMPLE DATE } & $02 / 24 / 92$ & $05 / 26 / 82$ & $08 / 21 / 92$ & $11 / 16 / 92$ & \\
\hline \multicolumn{7}{|l|}{ FIELD DATA } \\
\hline \multicolumn{2}{|c|}{ Andrte } & 1092 & 20992 & 3092 & 4092 & Unit \\
\hline \multicolumn{2}{|c|}{$\begin{array}{l}\text { Water elevation } \\
\text { pH } \\
\text { Sp. conductance } \\
\text { Water temperature } \\
\text { Alkalinity as } \mathrm{CaCO}_{3} \\
\text { Volume purged }\end{array}$} & $\begin{array}{l}269.3 \\
4.1 \\
155 \\
22.8 \\
0 \\
5.1\end{array}$ & $\begin{array}{l}269.3 \\
4.3 \\
143 \\
23.0 \\
0 \\
5.1\end{array}$ & $\begin{array}{l}271.2 \\
5.0 \\
151 \\
22.9 \\
0 \\
3.9\end{array}$ & $\begin{array}{l}270.6 \\
5.4 \\
232 \\
22.0 \\
8 \\
0.9\end{array}$ & $\begin{array}{l}\text { ft mal } \\
\mathrm{pH} \\
\mu \mathrm{S} / \mathrm{cm} \\
{ }^{\circ} \mathrm{C} \\
\mathrm{mg} / \mathrm{L} \\
\text { Well vol. }\end{array}$ \\
\hline
\end{tabular}

ANALYTICAL DATA

H D Analue

Arsenic

Barium

Benzene

Bromodichloromethane

Bromoform

Bromomethane (Methyl bromide)

Cadmium

Calcium

Carbon tetrachloride

Chloride

Chlorobenzeno

Chloroothane

Chloroethene (Vinyl chloride)

2-Chloroethyl vinyl other

Chloroform

Chloromothane (Mothyl chloride)

Chromium

Dibromochloromothane

1.1-Dichloroethane

1,2-Dichloroethane

1,1-Dichloroethylene

trans-1,2-Dichloroothylone

Dichloromethane (Methylene chloride)

2,4-Dichlorophenoxyacetic acid

1,2-Dichioropropane

cis-1,3-Dichloropropene

trans-1,3-Dichloropropene

Endrin

Ethylbenzene

Fluoride

Grose alpha

Iron

Lead

Lindane

Magnesium

Manganese

Morcury

Methoxychlor

Nitrate as nitrogen

Nonvolatile beta

pH

Phonole

Potassium

1092

$\underline{2092}$

$\underline{3092}$

$<2.0$
$<6.6$

$<2.0$

4.0

$<5.0$

$<5.0$

$<10$

0.35

154

3,150

122

$<5.0$

7.180

$<5.0$

$<10$

$<10$

$<5.0$

$<10$

$<1.1$

1.1

$<5.0$

$<5.0$

$<5.0$

$<5.0$

$<5.0$

1.3
$<1.1$

$<1.1$
$<5.0$

$<5.0$

$<5.0$

$<0.11$

$<0.11$

$<5.0$

$<100$

$<100$

$<1.1 E+\infty 0$

$<3.0 E+\infty 0$

182
4.6

4.6
$<0.056$

126

6.1

$<0.20$

$<0.20$
$<0.56$

1,970

2. $2 E+00$

290

11
$<0.056$

$<0.056$

113
7.2

$<0.20$

$<0.20$
$<0.56$

1,900

$<5.0 E+00$

5.3

5.6

$<5.0$

$<5.0$
225

186

$<2.0$

Mod

Unit Lab Flag

$<4.0$

$<0.35$

361

3,550

$$
\begin{aligned}
& 0.35 \\
& 146
\end{aligned}
$$

3,090

$\begin{array}{lll}\mu g / L & \text { WA } & 0 \\ \mu g / L & \text { WA } & 0\end{array}$

$\mu g / L$

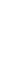

$\mu \sigma / L$

$\mu g / L$

WA 0

WA 0

WA 0

o/l

$\mu \mathrm{g} / \mathrm{L}$

$\mu \mathrm{g} / \mathrm{L}$

$\mu \sigma / L$

$\mu \mathrm{g} / \mathrm{L}$

$$
<1 \text {. }
$$

$<1.1$

v

WA 0

$\mu g / L$

$\mu \mathrm{g} / \mathrm{L}$

$\mu \mathrm{g} / \mathrm{L}$

$\mu \mathrm{g} / \mathrm{L}$

$\mu g / L$

$<1.1<1$

1.1

$\mu \mathrm{g} / \mathrm{L}$

WA 0

$<0.11<0.10$

$<100<100$

$<2.0 E+\infty 0$

321

3.7

$<0.056$

179

9.1

$<0.20$

$<0.56$

1.060

$<2.0 E+00$

5.4

5.4
5.0

$<2.0 E+00$

716

10

$<0.052$

106

21

$<0.20$

$<0.52$

1.250

$<2.0 \mathrm{E}+00$

5.7

$<5.0$

243

$\mu g / L$

$\mu g / L$ WA 0

$\mu \mathrm{g} / \mathrm{L}$

$\mu \mathrm{g} / \mathrm{L}$ WA 0

$\mathrm{PCi} / \mathrm{L}$ CN $\mathrm{O}$

$\mu g / L$ WA 2

$\mu g / L$ WA

$\mu g / L$ WA 0

$\mu g / L$ WA

HA WA

$\mu g / L$ WA 0

$\begin{array}{lll}\mu \boldsymbol{g} / \mathrm{L} & \text { WA } & 0 \\ \mu \mathrm{g} / \mathrm{L} & \text { WA } & 0\end{array}$

$\begin{array}{lll}\mu \mathrm{g} / \mathrm{L} & \text { WA } & 0 \\ \mathrm{pCi} / \mathrm{L} & \mathrm{CN} & 0\end{array}$

$\begin{array}{llll}\mathrm{pCi} / \mathrm{L} & \mathrm{CN} & 0 \\ \mathrm{pH} & \text { WA } & 0\end{array}$

J3 $\quad \begin{array}{llll}\mu g / L & \text { WA } & 0 \\ \mu g / L & \text { WA } & 0\end{array}$

Note: Flagging levels, modifiers, and laboratories are for 4th quarter 1992 data only. See Appendix B for flagging criteria.

- = exceeded holding time for 4th quarter 1992.

- =xceeded final primary drinking water standard for 4th quarter 1992. 
Well HAC 1 continued

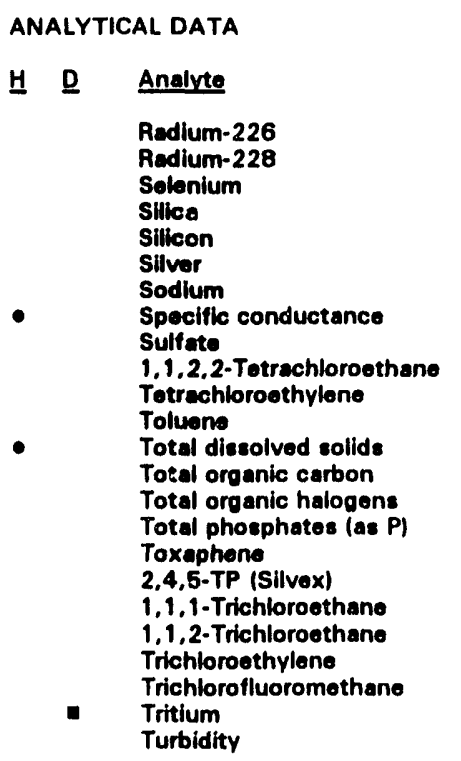

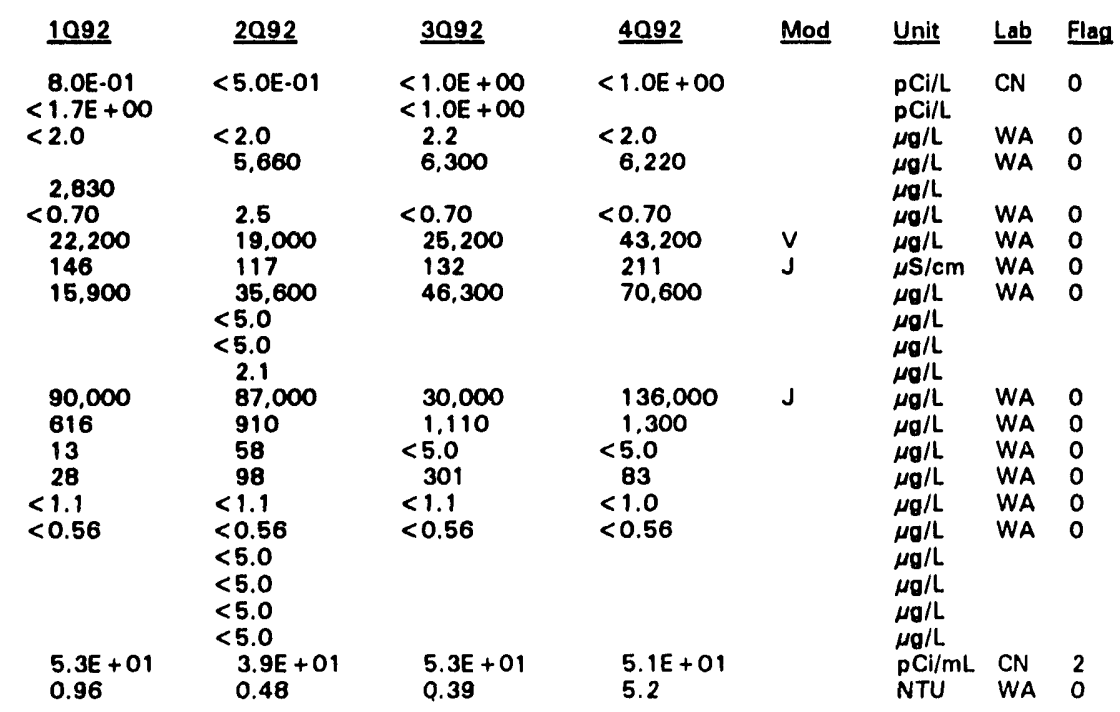

\section{WELL HAC 2}

$\begin{array}{ll}\text { SRS Coord. } & \text { Lat/Lonqitude } \\ \text { N72220.2 } & 33.285629{ }^{\circ} \mathrm{N} \\ \text { E61366.9 } & 81.645495{ }^{\circ} \mathrm{W} \\ & \end{array}$

\section{Screen Zone Elevation \\ 278.8-258.8 ft msl}

$02 / 24 / 92$

FIELD DATA

\begin{tabular}{l}
1092 \\
\hline 268.8 \\
5.0 \\
405 \\
22.0 \\
12 \\
0.5
\end{tabular}

1092
$<2.0$
9.7
0.45
315
3.720
$<1.1$
$<1.1$
$<0.11$
$<100$
$<1.9 \mathrm{E}+00$
95
$<2.0$
$<0.056$
451

\begin{tabular}{ll} 
Top of Casing & \\
$298.1 \mathrm{tt} \mathrm{m8l}$ & \\
& \\
$05 / 26 / 92$ & $08 / 21 / 9$ \\
& \\
20.92 & 3092 \\
\hline 268.8 & 270.8 \\
5.3 & 5.5 \\
521 & 426 \\
21.8 & 22.0 \\
5 & 13 \\
0.8 & 0.9
\end{tabular}

\section{Casing}

4" PVC

$\frac{\text { Pump }}{\mathrm{s}}$

Formation

Water table

ANALYTICAL DATA

H D Analyte
Arsenic
Barium
Cadmium
Calcium
Chloride
Chromium
2,4-Dichlorophenoxyacetic acid
Endrin
Fluoride
Grose alpha
lron
Lead
Lindane
Magnesium

$\begin{array}{ll}2092 & \underline{3092} \\ <2.0 & <2.0 \\ 8.6 & 5.5 \\ 1.2 & 0.92 \\ 300 & 283 \\ 7.510 & 4.490 \\ <1.1 & <1.1 \\ <1.1 & <1.1 \\ <0.11 & <0.11 \\ <100 & <100 \\ <3.0 \mathrm{E}+00 & <2.0 \mathrm{E}+00 \\ 50 & 50 \\ <2.0 & <2.0 \\ <0.056 & <0.056 \\ 387 & 247\end{array}$

$\quad \underline{4092}$
$<2.0$
9.3
0.67
259
5.480
1.4
$<1.2$
$<0.12$
$<100$
$2.3 E+00$
2.070
6.0
$<0.059$
375

\begin{tabular}{|c|c|c|}
\hline Mod & Unit & Lab \\
\hline & $\mu g / L$ & WA \\
\hline J3 & $\mu \mathrm{g} / \mathrm{L}$ & WA \\
\hline \multirow[t]{3}{*}{ J3 } & $\mu \mathrm{g} / \mathrm{L}$ & WA \\
\hline & $\mu \mathrm{g} / \mathrm{L}$ & WA \\
\hline & $\mu \mathrm{g} / \mathrm{L}$ & WA \\
\hline \multirow[t]{5}{*}{ J3 } & $\mu \mathrm{g} / \mathrm{L}$ & WA \\
\hline & $\mu \mathrm{g} / \mathrm{L}$ & WA \\
\hline & $\mu \mathrm{g} / \mathrm{L}$ & WA \\
\hline & $\mu \mathrm{g} / \mathrm{L}$ & WA \\
\hline & $\mathrm{pCi} / \mathrm{L}$ & $\mathrm{CN}$ \\
\hline V & $\mu \mathrm{g} / \mathrm{L}$ & WA \\
\hline J3 & $\mu \mathrm{g} / \mathrm{L}$ & WA \\
\hline & $\mu \mathrm{g} / \mathrm{L}$ & WA \\
\hline V & & WA \\
\hline
\end{tabular}

\footnotetext{
Note: Flagging levels, modifiers, and laboratories are for 4th quarter 1992 data only. See Appendix B for flagging criteria. - = exceeded holding time for 4th quarter 1992.

- exceeded final primary drinking water standard for 4th quarter 1992.
} 
Well HAC 2 continued

ANALYTICAL DATA

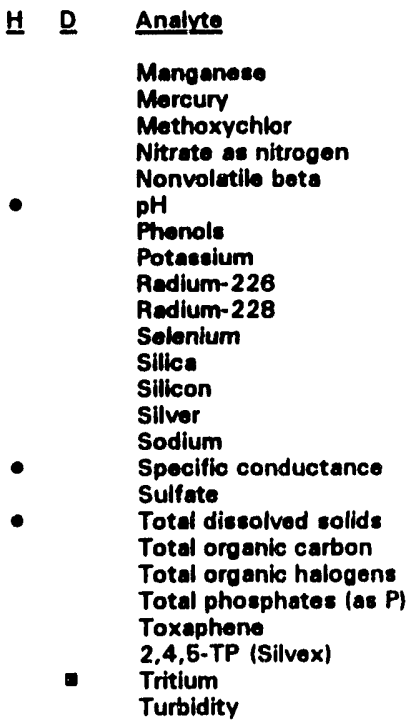

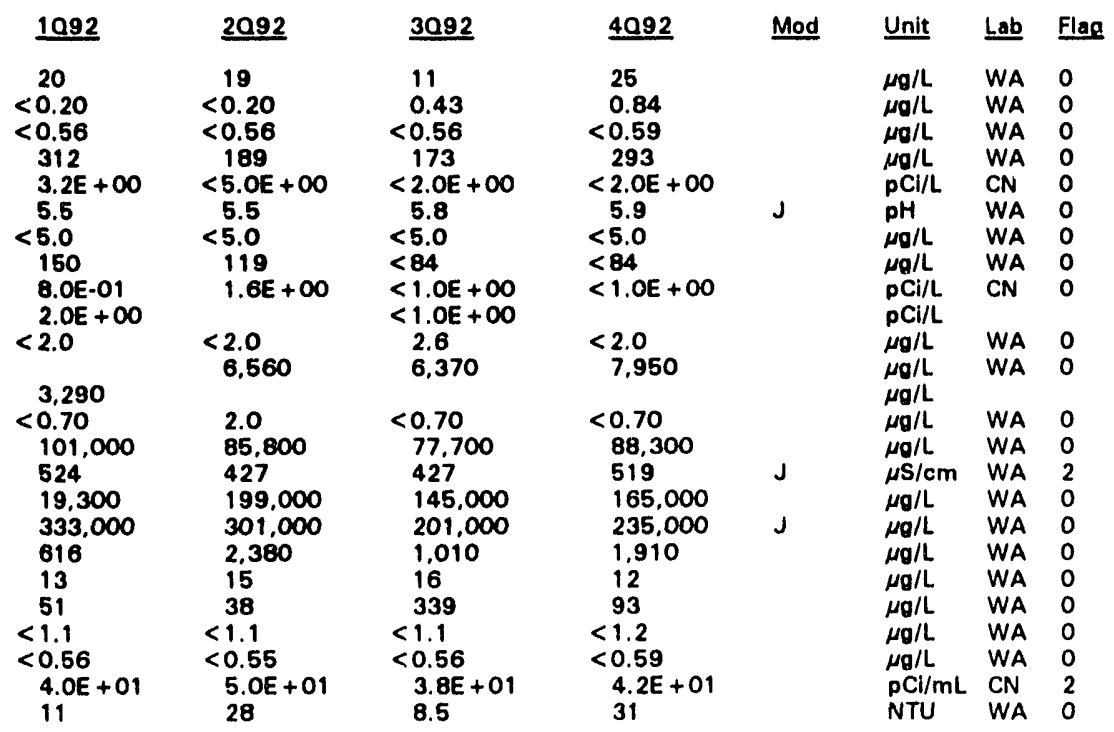

\section{WELL HAC 3}

\section{SRS Coord. Las/Longitude \\ N72183.4 \\ E61313.6 \\ 33.285461 o $N$ $81.645664{ }^{\circ} \mathrm{W}$}

SAMPLE DATE

FIELD DATA

\section{Screen Zone Elovation \\ 275.0-255.0 $\mathrm{ft} \mathrm{msl}$}

$02 / 24 / 92$
Top of Casing

298 ft msl

05/26/92

2092
269.1
4.8
220
22.1
0
0.9

$\begin{array}{ll} & 1092 \\ < & 2.0 \\ 14 \\ 0.42 \\ 330 \\ 6.480 \\ <1.1 \\ <1.1 \\ <0.11 \\ <100 \\ 3.6 \mathrm{E}+00 \\ 81 \\ 2.4 \\ <0.056\end{array}$

\section{0}

\begin{tabular}{ll}
$\underline{2092}$ & $\underline{3092}$ \\
\hline$<2.0$ & $<2.0$ \\
16 & 8.3 \\
0.67 & 0.92 \\
406 & 2.490 \\
11.200 & 10.300 \\
$<1.1$ & $<1.1$ \\
$<1.1$ & $<1.1$ \\
$<0.11$ & $<0.11$ \\
$<100$ & $<100$ \\
$<3.0 E+00$ & $<2.0 E+00$ \\
90 & 96 \\
6.8 & 2.2 \\
$<0.057$ & $<0.055$
\end{tabular}

4" PVC

$08 / 21 / 92$

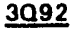

271.0

5.5

23.

28
1.0

ANALYTICAL DATA

H D

Analyte
Arsenic
Barium
Cadmium
Calcium
Chloride
Chromium
2,4-Dichlorophenoxyacetic acid
Endrin
Fluoride
Grose alpha
Iron
Lead
Lindane

92
1.0
5
6.2
0

4092
270.5
5.4
375
21.4
11
0.8

Unit
$\mathrm{ft} \mathrm{msl}$
$\mathrm{pH}$
$\mu \mathrm{S} / \mathrm{cm}$
${ }^{\circ} \mathrm{C}$
$\mathrm{mg} / \mathrm{L}$
Well vol.

$\begin{aligned} & 4092 \\ &< 2.0 \\ & 8.4 \\ & 0.38 \\ & 918 \\ & 10.700 \\ & 2.4 \\ &< 1.2 \\ &< 0.11 \\ &< 100 \\ &< 2.0 E+00 \\ & 406 \\ & 13 \\ &< 0.053\end{aligned}$

\begin{tabular}{|c|c|c|}
\hline Mod & Unit & $\underline{\text { Lab }}$ \\
\hline & $\mu \mathrm{g} / \mathrm{L}$ & WA \\
\hline J3 & $\mu \mathrm{g} / \mathrm{L}$ & WA \\
\hline V & $\mu \mathrm{g} / \mathrm{L}$ & WA \\
\hline & $\mu g / L$ & WA \\
\hline & $\mu \mathrm{g} / \mathrm{L}$ & WA \\
\hline V & $\mu \mathrm{g} / \mathrm{L}$ & WA \\
\hline & $\mu \mathrm{g} / \mathrm{L}$ & WA \\
\hline & $\mu \mathrm{g} / \mathrm{L}$ & WA \\
\hline & $\mu \mathrm{g} / \mathrm{L}$ & WA \\
\hline & $\mathrm{pCl} / \mathrm{L}$ & $\mathrm{CN}$ \\
\hline & $\mu \boldsymbol{g} / L$ & WA \\
\hline & $\mu \boldsymbol{g} / \mathrm{L}$ & WA \\
\hline & $\mu_{\mathrm{g}} / \mathrm{L}$ & WA \\
\hline
\end{tabular}

Note: Flagging levels, modifiers, and laboratories are for 4th quarter 1992 data only. See Appendix B for flagging criteria.

- =xceeded holding time for 4th quarter 1992.

= exceeded final primary drinking water standard for 4th quarter 1992. 


\section{ANALYTICAL DATA}

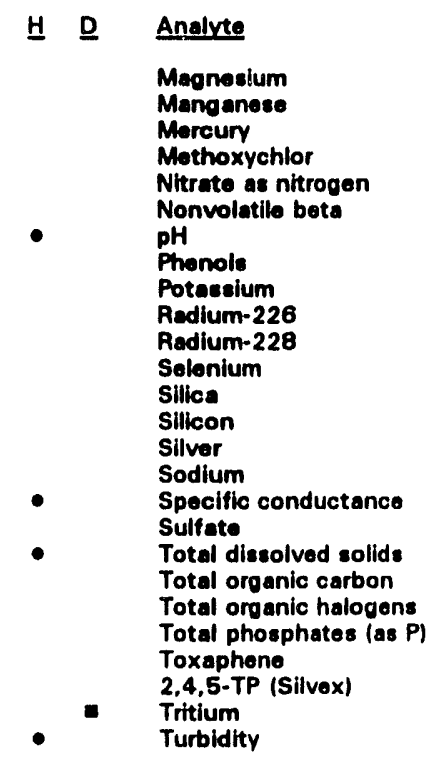

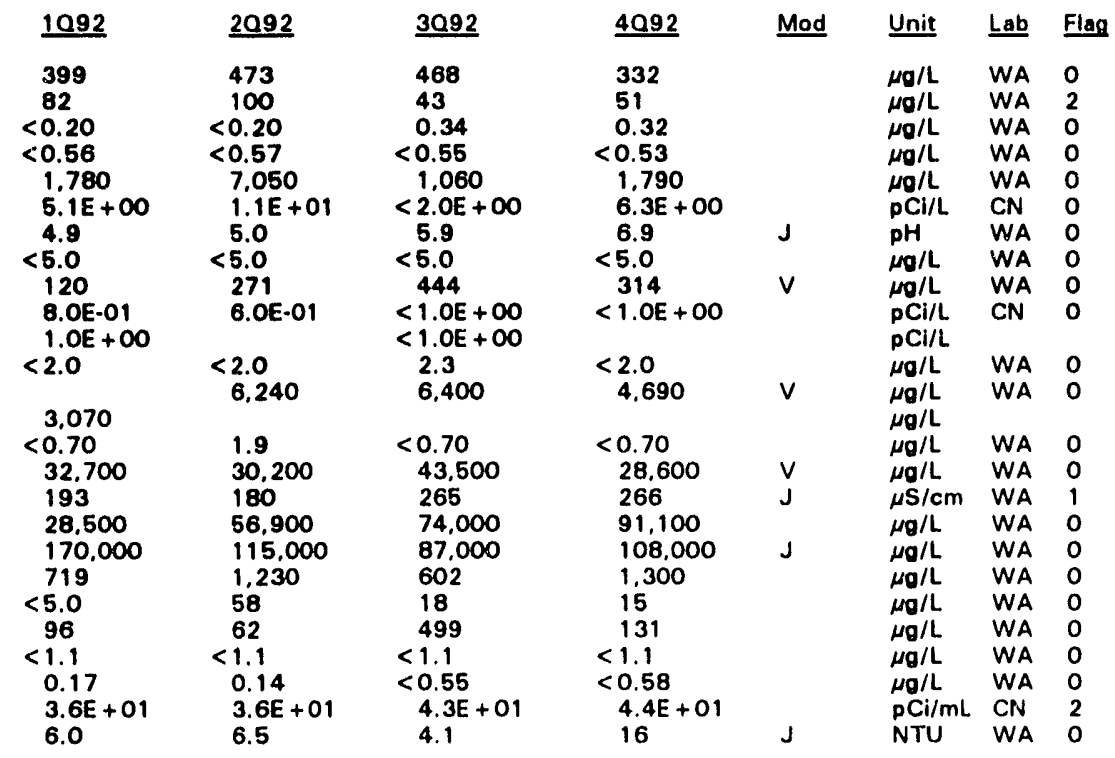

\section{WELL HAC 4}

\section{SAS Coord. Lat/Longitude
N721 20.3
33.285416 o $N$ \\ E61372.0 $81.645287{ }^{\circ} \mathrm{W}$}

\begin{abstract}
Scroen Zone Elovation
274.1-254.1 ft mst
\end{abstract}

$02 / 24 / 92$

\section{Top of Casing}

$296.9 \mathrm{ft} \mathrm{msl}$
Casing
4" PVC

Pump

Formation

SAMPLE DATE$$
02 / 24 / 92
$$

$05 / 26 / 92$

$08 / 21 / 92$

$11 / 18 / 92$

FIELD DATA

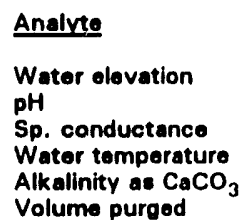

1092
269.5
4.3
48
22.7
0
4.0

2092
269.5
4.6
43
22.8
0
4.0

3092
271.4
4.6
36
23.2
0
4.0

$\begin{array}{ll}\frac{1092}{10} & \underline{2092} \\ <2.0 & <2.0 \\ 12 & 10 \\ <0.35 & 0.42 \\ 57 & 76 \\ 2.480 & 4.770 \\ <1.1 & 2.6 \\ <1.1 & <1.1 \\ <0.11 & <0.11 \\ <100 & <100 \\ 1.1 \mathrm{E}+\infty & <3.0 \mathrm{E}+00 \\ 23 & 42 \\ 4.7 & 7.0\end{array}$

$\quad 3092$
$<2.0$
7.9
0.92
124
3.790
$<1.1$
$<0.30$
$<0.0060$
$<100$
$<2.0 \mathrm{E}+00$
29
5.6

$\frac{4092}{<}$
$<2.0$
7.5
1.3
57
3.400
2.7
$<1.1$
$<0.11$
$<100$
$<2.0 \mathrm{C}+00$
24
4.9

Mod
J3
J3
$\mathrm{V}$
J3

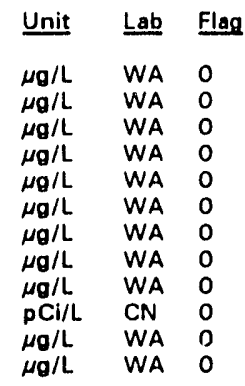

ANALYTICAL DATA

Andyte
Arsenic
Barium
Cadmium
Calcium
Chlorido
Chromium
2,4-Dichlorophenoxyacetic acid
Endrin
Fluoride
Grose alpha
Iron
Load

23
4.7

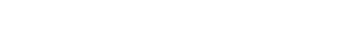


Well HAC 4 continued

\section{ANALYTICAL DATA}

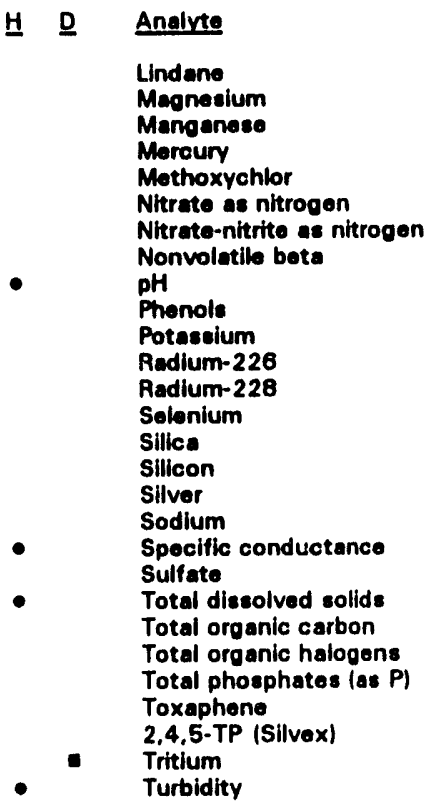

$\begin{array}{lcl}1092 & \underline{2 Q 92} & \underline{3092} \\ <0.056 & <0.057 & <0.0050 \\ 304 & 299 & 261 \\ 37 & 35 & 29 \\ <0.20 & <0.20 & <0.20 \\ <0.56 & <0.57 & <0.50 \\ 2.040 & 1.970 & 1.240 \\ 3.9 E+00 & <5.0 E+00 & 1.980 \\ 4.9 & 5.0 & 2.4 E+00 \\ <5.0 & <5.0 & 8.2 \\ 167 & 189 & <5.0 \\ <3.0 E-01 & <5.0 E-01 & <1.0 E+00 \\ <1.9 E+00 & <2.0 & 1.1 E+01 \\ <2.0 & 5.350 & <2.0 \\ & & 5.700 \\ 2.840 & 3.1 & 0.95 \\ <0.70 & 4.750 & 6.520 \\ 5.970 & 38 & 41 \\ 39 & <2,500 & 3.450 \\ <2.500 & 31.000 & 41.000 \\ 80.000 & 1.120 & <500 \\ 616 & <13 & 7.4 \\ <5.0 & <20 & 891 \\ <20 & <1.1 & <0.24 \\ <1.1 & <0.57 & <0.090 \\ <0.56 & 3.0 E+01 & 4.1 E+01 \\ 2.7 E+01 & 0.47 & 0.58 \\ 0.62 & & \\ & & \end{array}$

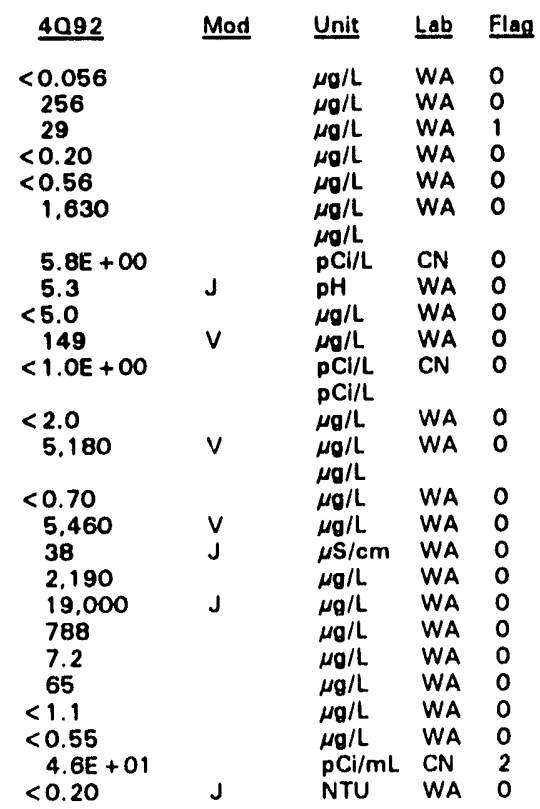

Note: Flagging levels, modifiers, and laboratories are for 4th quarter 1992 data only. See Appendix B for flagging criteria. - = exceeded holding time for 4th quarter 1992.

- exceeded final primary drinking water standard for 4th quarter 1992. 
Appendix E - Data Quality/Useability Assessment 


\section{Data Quality/Useability Assessment}

Quality assurance/quality control (QA/QC) procedures relating to accuracy and precision of analyses performed on groundwater samples are followed in the field and laboratory and are reviewed prior to publication of results. The Environmental Protection Department/ Environmental Monitoring Section's (EPD/EMS) review of the volume of analytical data acquired each quarter and presented in various reports is an ongoing process; its review of the QA/QC data cannot be completed in time to meet the deadlines for the reports required by the Resource Conservation and Recovery Act and associated regulations. Other site and regulatory personnel can obtain further information on the data quality and useability in a variety of ways, including those described below.

\section{Data Qualification}

The contract laboratories continually assess their own accuracy and precision according to U.S. Environmental Protection Agency (EPA) guidelines. They submit sample- or batchspecific QA/QC information either at the same time as analytical results or in a quarterly summary. Properly defined and used result modifiers (also referred to as qualifiers) can be a key component in assessing data useability. Result modifiers designed by EPD/EMS and provided to the primary laboratories are presented in Appendix D.

\section{Assessment of Accuracy of the Data}

Accuracy, or the nearness of the reported result to the true concentration of a constituent in a sample, can be assessed in several ways.

A laboratory's general accuracy can be judged by analysis of results obtained from known samples. The non-radionuclide contract laboratories analyze commercial reference samples every quarter at EPD/EMS' request. The results of these analyses are presented in the EPD/EMS quarterly report, The Savannah River Site's Groundwater Monitoring Program. The primary laboratories also seek or maintain state certification by participating periodically in performance studies; reference samples and analysis of results are provided by EPA. Results of these studies also are published in the EPD/EMS quarterly reports.

Analysis of blanks provides a tool for assessing the accuracy of both sampling and laboratory analysis. Results for all field blanks for the quarter can be found in the EPD/EMS quarterly reports. Any field or laboratory blanks that exceeded established minimums are identified in the same reports, in tables associating them with groundwater samples analyzed in the same batches.

Surrogates, organic compounds similar in chemical behavior to the compounds of interest but not normally found in environmental samples, are used to monitor the effect of the matrix on the accuracy of analyses for organic parameters. For example, for analyses of volatile organics by EPA method 8240 , three surrogate compounds are added to all samples 
and blanks in each analytical batch. In analyses of semivolatile organics, three to four acid compounds and three to four base/neutral compounds are used. Other surrogates are used in pesticides analyses. Percent recoveries for surrogate analyses are calculated by laboratory personnel, reported to EPD/EMS, reviewed, and entered into the database, but they are not published. If recoveries are not within specified limits, the laboratory is expected to re-run the samples or attach result qualifiers to the data identifying the anomalous results.

Sample-specific accuracy for both organic and inorganic parameters can be assessed by examination of matrix spike/matrix spike duplicate results. A sample is analyzed unspiked to determine a baseline set of values. A second portion of sample is spiked with known concentrations of compounds appropriate to the analyses being performed, typically 5 volatile organic compounds for volatile organic analyses, 11 semivolatile compounds for semivolatiles, 6 pesticide compounds for pesticides, all metals for metals analyses, and a known quantity of cyanide for cyanide analysis. The percentage of the spike compound which is recovered, i.e., measured in excess of the value obtained for the unspiked sample, is a direct measure of analytical accuracy. EPA requires matrix spike/matrix spike duplicates to be run at least once per 20 samples of similar matrix.

Matrix spike/matrix spike duplicate results are reported to EPD/EMS but are not published. For organic compounds, according to EPA guidelines, no action is taken on the basis of matrix spike/matrix spike duplicate data alone (i.e., no result modifiers are assigned solely on the basis of matrix spike results); however, the results can indicate if a lab is having a systematic problem in the analysis of one or more analytes.

In the case of inorganic compounds, such as metals, the matrix spike sample analysis provides information about the effect of each sample matrix on the digestion and measurement methodology. Data qualifiers can be assigned on the basis of the percentage of spike recovery and are reported in the published results tables.

\section{Assessment of Precision}

Precision of the analyses, or agreement of a set of replicate results among themselves, is assessed through the use of duplicates (laboratory-initiated) and blind replicates (provided by EPD/EMS). The results of duplicate and replicate analyses are presented in the results tables of the first, second, and third quarter reports as multiple entries for an analyte under a single well heading. The results of replicate analyses are presented in the results tables in first, second, and third quarter reports as two separate sets of results for the same well. Duplicate and replicate results are not presented in fourth quarter reports; the results tables present instead only the highest result for each analyte for each quarter of the year.

The laboratories assess precision by calculating the relative percent difference, or RPD, for each pair of laboratory-initiated duplicate results. During 1992, at least one of the contract laboratories used a data qualifier (J3) to modify metals analyses when the RPD for laboratory duplicates was greater than $20 \%$. 
Additional statistical comparisons of laboratory duplicate and blind replicate results, both intra- and interlaboratory, are presented in the EPD/EMS quarterly reports. The calculation used for these reports is the MRD, or mean relative difference, which is similar to EPA's RPD except that the MRD provides a single value for all of the analyses of a particular compound, either inter- or intralaboratory, during one quarter. Because detection limits may vary among samples, the MRD requires calculation of a reference detection limit, which is the detection limit at the 90 th percentile of the array of limits in the population of all replicate and duplicate analyses for a given analyte during a particular quarter. The MRD is not method-specific.

\section{Method-Specific Accuracy and Precision}

The contract laboratories' EPA-approved laboratory procedures include QA/QC requirements as an integral part of the methods. Thus, knowledge of the method used in obtaining data is an important component of determining data useability. EPA has conducted extensive research and development on the methods approved for the analysis of water and waste water; information on the accuracy and precision of the method is available from EPA publications, as is full information on required $\mathrm{QA} / \mathrm{QC}$ procedures. A listing of the methods used by the primary laboratories during first quarter 1992 is given below along with the source for the method description. Many, if not all, of these sources include presentations of representative accuracy and precision results.

\begin{tabular}{|c|c|c|}
\hline Method & Used to Analyze & Source \\
\hline EPA120.1 & Specific conductance & EPA EMSL 1983 \\
\hline EPA150.1 & pH & EPA EMSL 1983 \\
\hline EPA 160.1 & Filterable residue (total dissolved solids) & EPA EMSL 1983 \\
\hline EPA 160.2 & Nonfilterable residue & EPA EMSL 1983 \\
\hline EPA 180.1 & Turbidity & EPA EMSL. 1983 \\
\hline EPA200.7 & Trace elements & EPA EMSL 1983 \\
\hline EPA206.2 & Arsenic & EPA EMSL 1983 \\
\hline EPA208.2 & Barium & EPA EMSL 1983 \\
\hline EPA239.2 & Lead & EPA EMSL 1983 \\
\hline EPA245.1 & Mercury & EPA EMSL 1983 \\
\hline EPA270.2 & Selenium & EPA EMSL 1983 \\
\hline EPA279.2 & Thallium & EPA EMSL 1983 \\
\hline EPA300.0 & Inorganics, non-metallics & EPA EMSL 1991 \\
\hline EPA310.1 & Alkalinity & EPA EMSL 1983 \\
\hline EPA325. 2 & Chloride & EPA EMSL 1983 \\
\hline EPA335.3 & Cyanide & EPA EMSL 1983 \\
\hline EPA340.2 & Fluoride & EPA EMSL 1983 \\
\hline EPA353.1 & Nitrogen, nitrate-nitrite & EPA EMSL 1983 \\
\hline EPA353.2 & Nitrogen, nitrate, nitrite, or combined & EPA EMSL 1983 \\
\hline EPA353.3 & Nitrogen, nitrate-nitrite, or nitrite only & EPA EMSL 1983 \\
\hline EPA354.1 & Nitrogen, nitrite & EPA EMSL 1983 \\
\hline EPA365.1 & Phosphorus, all forms (reported as total phosphates) & EPA EMSL 1983 \\
\hline EPA365.2 & Phosphorus, all forms (reported as total phosphates) & EPA EMSL 1983 \\
\hline EPA375.4 & Sulfate, turbidimetric & EPA EMSL 1983 \\
\hline EPA376.2 & Sulfide & EPA EMSL 1983 \\
\hline APHA403 & Alkalinity & APHA 1985 \\
\hline EPA4 13.1 & Oil \& grease & EPA EMSL 1983 \\
\hline APHA4 15A & lodine & APHA 1985 \\
\hline
\end{tabular}




$\begin{array}{ll}\text { Method } & \text { Used to Analyze } \\ \text { EPA415.1 } & \text { Total organic carbon } \\ \text { EPA418.1 } & \text { Petroleum hydrocarbons } \\ \text { EPA420.1 } & \text { Phenolics } \\ \text { EPA420.2 } & \text { Phenolics } \\ \text { APHA705 } & \text { Total alpha-emitting radiurn } \\ \text { ASTMD3869C } & \text { lodide } \\ \text { APHA5320 } & \text { Dissolved organic halogen } \\ \text { EPA6010 } & \text { Metals } \\ \text { EPA7041 } & \text { Antimony } \\ \text { EPA7060 } & \text { Arsenic } \\ \text { EPA7421 } & \text { Lead } \\ \text { EPA7470 } & \text { Mercury } \\ \text { EPA7740 } & \text { Selenium } \\ \text { EPA7841 } & \text { Thailium } \\ \text { EPA8010 } & \text { Halogenated volatile organics } \\ \text { EPA8020 } & \text { Aromatic volatile organics } \\ \text { EPA8080 } & \text { Organochlorine pesticides and PCBs } \\ \text { EPA8140 } & \text { Organophosphorus pesticides } \\ \text { EPA8150 } & \text { Chlorinated herbicides } \\ \text { EPA8240 } & \text { GCMS VOA } \\ \text { EPA8270 } & \text { GCMS semivolatiles } \\ \text { EPA8280 } & \text { Dioxins and furans } \\ \text { EPA9012 } & \text { Total cyanide } \\ \text { EPA9020 } & \text { Total organic halides } \\ \text { EPA9030 } & \text { Sulfides } \\ & \\ & \end{array}$

\section{Source}

EPA EMSL 1983

EPA EMSL 1983

EPA EMSL 1983

EPA EMSL 1983

APHA 1985

ASTM 1992

APHA 1989

EPA 1986

EPA 1986

EPA 1986

EPA 1986

EPA 1986

EPA 1986

EPA 1986

EPA 1986

EPA 1986

EPA 1986

EPA 1986

EPA 1986

EPA 1986

EPA 1986

EPA 1986

EPA 1986

EPA 1986

EPA 1986

An example of the available method-specific QA/QC information is that for the analysis of metals by EPA Method 6010/200.7 (EPA 1986/EPA EMSL 1983). The primary laboratories, General Engineering Laboratories (GE) and Roy F. Weston, Inc. (Weston), use this inductively coupled plasma (ICP) atomic emission spectrometric method.

The following precision and accuracy data are based on the experience of seven laboratories that applied the ICP technique to acid-distilled water matrices that had been dosed with various metal concentrates. (Note: not all seven laboratories analyzed all 14 elements.) The references give results for samples having three concentration ranges; the results here are for samples having the lowest values, similar to actual groundwater results for SRS.

\section{ICP Precision and Accuracy Data}

\section{Element}

Beryllium

Manganese

Vanadium

Arsenic

Chromium

Copper

Iron

Aluminum

\section{True value $(\mu \mathrm{g} / \mathrm{L})$}

$$
20
$$

15

70

22

10

11

20

60
Mean reported value $(\mu \mathrm{g} / \mathrm{L}$ )

20

15

69

19

10

11

19

62
Mean percent

RSD $^{a}$

9.8

6.7

2.9

23

18

40

15

33 


\begin{tabular}{|c|c|c|c|}
\hline Element & True value $(\mathrm{kg} / \mathrm{L})$ & $\begin{array}{l}\text { Mean reported } \\
\text { value }(\mathrm{mg} / \mathrm{L})\end{array}$ & $\begin{array}{l}\text { Mean percent } \\
\underline{\mathrm{RSD}}^{\mathrm{a}}\end{array}$ \\
\hline Cadmium & 2.5 & 2.9 & 16 \\
\hline Cobalt & 20 & 20 & 4.1 \\
\hline Nickel & 30 & 28 & 11 \\
\hline Lead & 24 & 30 & 32 \\
\hline Zinc & 16 & 19 & 45 \\
\hline Selenium & 6 & 8.5 & 42 \\
\hline
\end{tabular}

Note: In EPA (1986), the column heading is Mean Standard Deviation (\%).

a Relative standard deviation.

As another example, EPA Method 601/8010 (EPA 1991a/EPA 1986) is used by both GE and Weston for analyses of halogenated volatile organics. In the presentation of the method in both references, the following table gives method-specific accuracy and precision as functions of concentration. Contract laboratories are expected to achieve or at least approach these limits.

Accuracy and Precision as Functions of Concentration for EPA Method 601/8010

\section{Parameter}

Bromodichloromethane
Bromoform
Bromomethane
Carbon tetrachloride
Chlorobenzene
Chloroethane
2-Chloroethyl vinyl ether
Chloroform
Chloromethane
Dibromochloromethane
1,2-Dichlorobenzene
1,3-Dichlorobenzene
1,4-Dichlorobenzene
1,1-Dichloroethane
1,2-Dichloroethane
1,1-Dichloroethene
trans-1,2-Dichloroethene
1,2-Dichloropropane
cis-1,3-Dichloropropene
trans-1,3-Dichloropropene
Methylene chloride
1,1,2,2-Tetrachlorethane
Tetrachloroethylene
1,1,1-Trichloroethane
1,1,2-Trichloroethane
Trichloroethylene

Accuracy as recovery, $X^{\prime 8}(\mathrm{mg} / \mathrm{L})$

$1.12 C-1.02^{d}$

$0.96 C-2.05$

$0.76 C-1.27$

$0.98 C-1.04$

$1.00 C-1.23$

$0.99 C-1.53$

$1.00 \mathrm{C}$

$0.93 C-0.39$

$0.77 C+0.18$

$0.94 C+2.72$

$0.93 C+1.70$

$0.95 C+0.43$

$0.93 C-0.09$

$0.95 C-1.08$

$1.04 C-1.06$

$0.98 C-0.87$

$0.97 C-0.16$

$1.00 \mathrm{C}$

$1.00 \mathrm{C}$

$1.00 \mathrm{C}$

$0.91 C-0.93$

$0.95 C+0.19$

$0.94 C+0.06$

$0.90 C-0.16$

$0.86 C+0.30$

$0.87 C+0.48$
Single analyst precision $(u \mathrm{~g} / L)^{b}$

$0.11 \bar{x}+0.04^{\circ}$

$0.12 \bar{x}+0.58$

$0.28 \bar{X}+0.27$

$0.15 \bar{X}+0.38$

$0.15 \bar{X}-0.02$

$0.14 \bar{X}-0.13$

$0.20 \bar{x}$

$0.13 \bar{x}+0.15$

$0.28 \bar{x}-0.31$

$0.11 \bar{x}+1.10$

$0.20 \bar{x}+0.97$

$0.14 \bar{x}+2.33$

$0.15 \bar{X}+0.29$

$0.09 \bar{x}+0.17$

$0.11 \bar{x}+0.70$

$0.21 \bar{x}-0.23$

$0.11 \bar{x}+1.46$

$0.13 \bar{x}$

$0.18 \bar{x}$

$0.18 \bar{x}$

$0.11 \bar{x}+0.33$

$0.14 \bar{x}+2.41$

$0.14 \bar{x}+0.38$

$0.15 \bar{x}+0.04$

$0.13 \bar{x}-0.14$

$0.13 \bar{x}-0.03$
Overall precision $(\mu \mathrm{g} / \mathrm{L})^{\mathrm{C}}$

$0.20 \bar{x}+1.00$

$0.21 \bar{x}+2.41$

$0.36 \bar{X}+0.94$

$0.20 \bar{x}+0.39$

$0.18 \bar{x}+1.21$

$0.17 \bar{X}+0.63$

$0.35 \bar{x}$

$0.19 \bar{x}-0.02$

$0.52 \bar{X}+1.31$

$0.24 \bar{X}+1.68$

$0.13 \bar{X}+6.13$

$0.26 \bar{X}+2.34$

$0.20 \bar{x}+0.41$

$0.14 \bar{X}+0.94$

$0.15 \bar{X}+0.94$

$0.29 \bar{X}-0.40$

$0.17 \bar{x}+1.46$

$0.23 \bar{x}$

$0.32 \bar{X}$

$0.32 \bar{x}$

$0.21 \bar{x}+1.43$

$0.23 \bar{x}+2.79$

$0.18 \bar{X}+2.21$

$0.20 \bar{x}+0.37$

$0.19 \bar{X}+0.67$

$0.23 \bar{x}+0.30$ 
Parameter

Trichlorofluoromethane

Vinyl chloride

Accuracy as
recovery, $X^{\prime a}(\mu g / L)$

$0.89 C-0.07$

$0.97 C-0.36$
Single analyst
precision $(\mu \mathrm{g} / \mathrm{L}$

$0.15 \bar{x}+0.67$

$0.13 \bar{x}+0.65$
Overall precision $(\mu \mathrm{g} / \mathrm{L})^{c}$

$0.26 \bar{x}+0.91$

$0.27 \bar{x}+0.40$

$\mathrm{X}^{\prime}=$ expected recovery for ane or more measurements of a sample containing a concentration of $C$, in $\mu g / L$.

b Experted single analyst standard deviation of measurements.

c Expected interlaboratory standard deviation of measurements.

d $C=$ true value for the concentration, in $\mu \mathrm{g} / \mathrm{L}$.

- $\bar{X}=$ average recovery found for measurements of samples containing a concentration of $C$, in $\mu \mathrm{g} / \mathrm{L}$.

Estimates based on performance in a single laboratory.

\section{References}

APHA (American Public Health Association), 1985. Standard Methods for the Examination of Water and Wasłewater, 16th edition. Washington, DC.

APHA (American Public Health Association), 1989. Standard Methods for the Examination of Water and Wastewater, 17th edition. Washington, DC.

ASTM (American Society for Testing and Materials), 1992. 1992 Annual Book of ASTM Standards, Volume 11.02, Water (II). Philadelphia, PA.

CFR (Code of Federal Regulations), 1991. Analytical Methods, Title 40, Part 136, Appendix A. Revised July 1, 1991. Washington, DC.

EPA (U.S. Environmentai Protection Ag!ncy), 1986. Test Methods for Evaluating Solid Waste (SW-846), Volumes IA-IC. Washington, DC.

EPA (U.S. Environmental Protection Agency), 1987. Data Quality Objectives for Remedial Response Activities. PB88-131870; EPA/540/G-87/003. Washington, DC.

EPA (U.S. Environmental Protection Agency), 1988a. Contract Laboratory Program Statement of Work for Inorganics Analysis, Multi-Media, Multi-Concentration. SOW No. 788. Washington, DC.

EPA (U.S. Environmental Protection Agency), 1988b. Contract Laboratory Program Statement of Work for Organics Analysis Multi-Media, Multi-Concentration. SOW No. 288. Washington, DC.

EPA (U.S. Environmental Protection Agency), 1990. Guidance for Data Useability in Risk Assessment. Interim Final. EPA/540/G-90/008. Washington, DC.

EPA EMSL (U.S. Environmental Protection Agency, Environmental Monitoring and Systems Laboratory), 1979. Handbook for Analytical Quality Control in Water Wastewater Laboratories. PB-297 151; EPA-600/4-79-019. Cincinnati, OH. 
EPA EMSL (U.S. Environmental Protection Agency, Environmental Monitoring and Systems Laboratory), 1983. Methods for Chemical Analysis of Water and Wastes. Revised March 1983. Cincinnati, $\mathrm{OH}$.

EPA EMSL (U.S. Environmental Protection Agency, Environmental Monitoring and Systems Laboratory), 1991. Test Method, The Determination of Inorganic Anions in Water by Ion Chromatography-Method 300.0. Revised August 1991. Cincinnati, OH. 

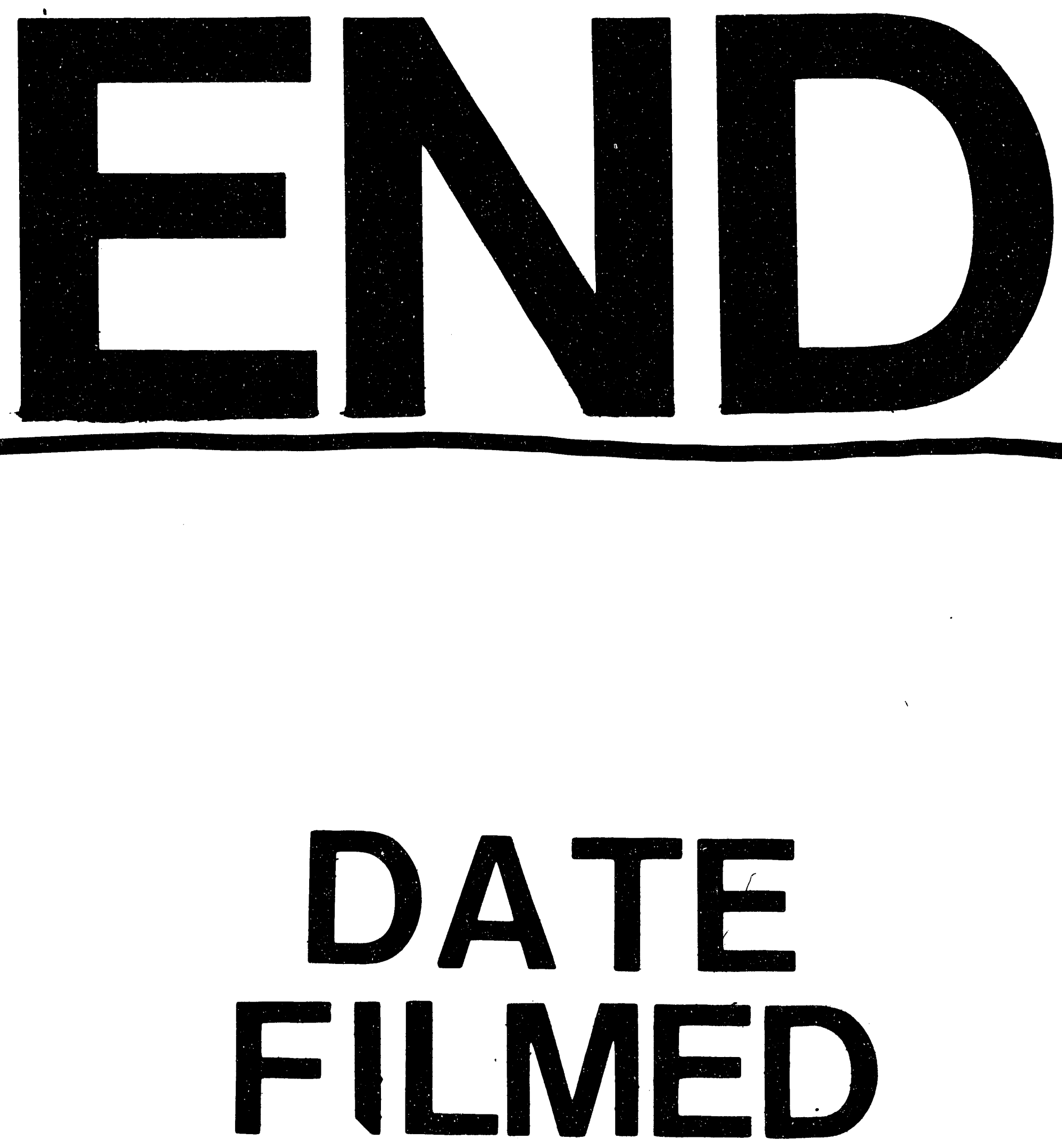

9
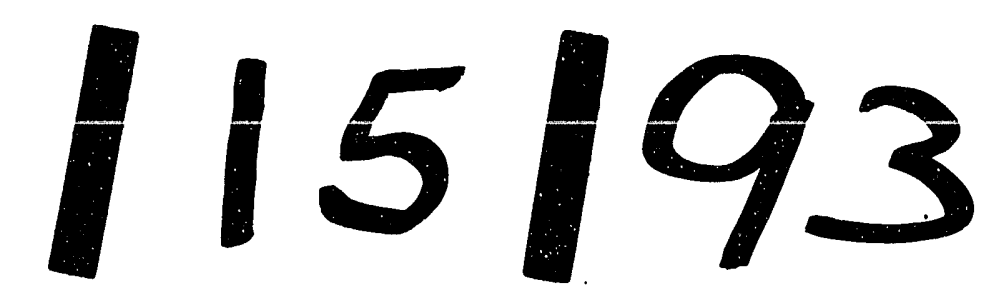
1 